\title{
Optimal Choice of When to Garbage Collect
}

\author{
NICHOLAS JACEK, MENG-CHIEH CHIU, BENJAMIN M. MARLIN, and \\ J. ELIOT B. MOSS, University of Massachusetts Amherst, USA
}

\begin{abstract}
We consider the ultimate limits of program-specific garbage collector (GC) performance for real programs. We first characterize the GC schedule optimization problem. Based on this characterization, we develop a linear-time dynamic programming solution that, given a program run and heap size, computes an optimal schedule of collections for a non-generational collector. Using an analysis of a heap object graph of the program, we compute a property of heap objects that we call their pre-birth time. This information enables us to extend the non-generational GC schedule problem to the generational GC case in a way that also admits a dynamic programming solution with cost quadratic in the length of the trace (number of objects allocated). This improves our previously reported approximately optimal result. We further extend the two-generation dynamic program to any number of generations, allowing other generalizations as well. Our experimental results for two generations on traces from Java programs of the DaCapo benchmark suite show that there is considerable promise to reduce garbage collection costs for some programs by developing program-specific collection policies. For a given space budget, optimal schedules often obtain modest but useful time savings, and for a given time budget, optimal schedules can obtain considerable space savings.
\end{abstract}

\section{CCS Concepts: • Software and its engineering $\rightarrow$ Runtime environments;}

Additional Key Words and Phrases: Automatic storage management, garbage collection, optimal schedules

\section{ACM Reference format:}

Nicholas Jacek, Meng-Chieh Chiu, Benjamin M. Marlin, and J. Eliot B. Moss. 2019. Optimal Choice of When to Garbage Collect. ACM Trans. Program. Lang. Syst. 41, 1, Article 3 (January 2019), 35 pages.

https://doi.org/10.1145/3282438

\section{INTRODUCTION}

Garbage collection (GC), i.e., automatic storage reclamation, has been a feature of some programming languages from the early days of high-level languages, and now it is expected for many major languages (and intentionally avoided by others). While GC technology has advanced greatly, reaching a stage of maturity where its overheads are acceptable for casual (and some heavy production) use as well as in real-time systems, we know little about the limits of its possible performance. There are a few worst-case bounds on explicit allocation and freeing schemes [12-14], for compacting GC [2], and for reference counting GC [5]. These bounds assume that one first fixes a storage management algorithm, and then presents it with a program whose allocation behavior the algorithm must carry out. Such bounds are proved by considering an adversarial program.

This work is supported by the National Science Foundation under grant CCF-1320498.

Authors' addresses: N. Jacek, M.-C. Chiu, B. M. Marlin, and J. E. B. Moss, University of Massachusetts Amherst, 140 Governors Dr., Amherst, MA, 01003; emails: \{njacek, joechiu, marlin, moss\}@cs.umass.edu.

Permission to make digital or hard copies of all or part of this work for personal or classroom use is granted without fee provided that copies are not made or distributed for profit or commercial advantage and that copies bear this notice and the full citation on the first page. Copyrights for components of this work owned by others than the author(s) must be honored. Abstracting with credit is permitted. To copy otherwise, or republish, to post on servers or to redistribute to lists, requires prior specific permission and/or a fee. Request permissions from permissions@acm.org.

(c) 2019 Copyright held by the owner/author(s). Publication rights licensed to ACM

0164-0925/2019/01-ART3 \$15.00

https://doi.org/10.1145/3282438 
In practice, however, we are generally concerned with how a storage management algorithm performs when presented with a particular program: the program we wish to run now. We can measure the performance of various GC algorithms on this program, but we have no idea how good they are in an absolute sense. Before an earlier version of this article appeared [8], there were no published results on the lowest possible GC cost achievable for a particular program through careful choice of when to collect. Li et al. [9] consider a GC optimization problem where the goal is minimizing the same cost measure we target, namely, GC effort (measured in bytes traced by the collector) per byte allocated. However, they work from an estimate of average behavior in a program, and they seek to choose a good heap size for a particular run of the program, i.e., the size beyond which additional space brings little performance benefit. They essentially assume what we call the default policy, i.e., to invoke the collector only when the heap is full. We seek to find, for each given heap size, the exact pattern of collections that will lead to minimal cost. In some cases this is quite different from what the default policy achieves. While the cost measures and the settings are similar, we are really solving a different problem, and we use different methods. We are not aware of any work more closely related to ours than this.

We seek here to fill the gap in knowledge of the lowest possible GC cost by developing and evaluating a methodology for assessing the limits of program-specific GC performance. Our earlier article described optimal GC schedules for a non-generational collector model, and approximately optimal ones for a generational model. Here we develop a technique for computing true optimal schedules for a generational collection scheme. We focus primarily on the two-generation case, but also give a solution (in Appendix B) for an arbitrary number of generations, which can also be generalized in some other ways. We had suspected that the generational collection schedule problem, like many optimal schedule problems, would be NP-complete. However, we were surprised to find an arguably realistic dynamic programming model whose solution costs $O\left(n^{2}\right)$ in the number of allocations for the two-generation case, and $O\left(n^{3}\right)$ for three or more generations.

What we present here is a limit study. Our goal is to determine the best possible GC performance (here measured in terms of bytes traced or copied). To accomplish this goal, we present methods for optimizing over the space of all possible sequences of decisions of when to collect that are consistent with not overflowing a given heap size. We refer to an optimal sequence of collection points as an optimal GC schedule.

When considering optimality of GC, we find it helpful to distinguish between an optimal schedule and an optimal policy. An optimal GC schedule is a GC schedule of lowest cost in a specific situation, e.g., for a specific heap size. Note that optimality of a GC schedule is defined with respect to a specific execution, i.e., a trace of a particular program executing in a particular situation. For a deterministic program and language implementation, an execution may be defined by the combination of a program and its input/environment. A schedule says nothing about how that schedule is obtained.

A GC policy, in contrast to a schedule, uses some information about the state of execution of the program, often some current and recent allocation and collection statistics, to determine when to collect. An optimal GC policy (for a particular execution) is one that leads to an optimal schedule. Note that a policy, as used here, is situation-specific. It need not perform well or even be applicable in other situations. In our larger research plan, we are interested in program-specific, but situation-general, policies, but here the focus is on the ultimate limits of performance, which can be characterized in terms of minimum cost schedules. These limits give a yardstick for measuring how well more general policies perform.

Again, we emphasize that our methods for finding an optimal schedule work entirely after the fact and offline. They do not lead directly to an optimal GC policy or algorithm. We also restrict ourselves in a number of ways that make the optimization problem more tractable, e.g., we consider 
only fixed heap sizes, not varying ones, and omit mechanisms such as pretenuring, large-object spaces, and so forth. For our cost model (bytes of objects traced by the collector), optimal cost is non-increasing as one increases the maximum heap size. Thus, there is no cost advantage to varying the heap size-one would just use the largest size one can. It may be possible to develop dynamic programming solutions for more complex memory management models, but their solution may be NP-complete. Even if their solution is not NP-complete, more complex models may result in polynomial time with a higher exponent, which can make exact solution prohibitively expensive in practice. (Here we generalize, in Appendix B, from two generations with $O\left(n^{2}\right)$ cost, to three or more generations, which has $O\left(n^{3}\right)$ cost for exact solution-worse if we consider the impact of having to search over various splits between the space used in one generation and the space used in another.)

To develop optimal GC schedules, we work from traces of allocation and heap behavior acquired using the Elephant Tracks (ET) tool $[10,11]$. We first consider the simplest case: non-generational (NG) GC with a fixed heap size. Since a given schedule is optimal only for a specific trace and heap size, we compute optimal schedules for a variety of heap sizes.

In principle, we could explore schedules that allow collection at any point in the trace, i.e., just before any allocation. For practicality, and to mirror more typical storage management behavior, we limit the points we consider to ones spaced about $256 \mathrm{~KB}^{1}$ apart, i.e., we assume a block-oriented scheme with a block size of $256 \mathrm{~KB} .^{2}$ Because we group objects together according to the block size, we use the term group size for this concept. We model NG and generational (Gen) GC as non-deterministic state machines, and then show that, given the information available from an ET trace after some simple post-processing, we can find their minimum cost schedules via dynamic programs. Our experimental results include exploration of the impact of using smaller group sizes, for cases for which the computation time was feasible. ${ }^{3}$

The baseline against which we compare is simple: the schedule produced by collecting only when the heap is full, i.e., when the next group of allocated objects will not fit. We call that the default policy. In all cases, we use as our measure of cost the volume of the objects traced by a tracing collector.

At this point we should mention that, because we use ET, which offers fine-grained analysis of when objects "die" (become unreachable), and ET is Java-specific, our results pertain to Java programs. Specifically, we use traces from programs in the DaCapo benchmark suite [3, 4], a suite designed to reveal various interesting storage use and GC behaviors.

After presenting results on NG GC, we turn to generational (Gen) GC. We use the same traces from Java programs, but we use some novel post-processing to compute a property of each object that we call the pre-birth time. We show that, given the pre-birth, birth, and death time, we can compute the cost of any GC schedule easily, without having to model the graph of heap objects. Put another way, the pre-birth time summarizes all we need to know, beyond the birth and death times of each object, to determine the cost of each collection in a GC schedule. We show that, as for NG GC, we can use a (different) dynamic program to find the lowest cost schedule for Gen GC.

In addition to offering exactly optimal results for Gen GC (and more), we go beyond our prior presentation by considering how optimal schedules offer space savings. While in many cases the time overhead of GC may already be acceptable (if GC costs $10 \%$ of execution time, and an optimal schedule would reduce GC time by $20 \%$, then that is only $2 \%$ of overall time saved), we find that

\footnotetext{
${ }^{1}$ We use $\mathrm{K}$ and $\mathrm{M}$ for powers of 1,024; B for bytes.

${ }^{2}$ We say "about $256 \mathrm{~KB}$ " because of the need to align on object boundaries, giving the specifics later.

${ }^{3}$ If average object size is 50 bytes, then an object-level trace is about 5,000 times longer than a group-level one. Given that actual solution costs are close to $O\left(n^{2}\right)$, the cost savings is a factor of more than $10^{7}$.
} 
optimal schedules often allow the same time performance in a much smaller heap. This may be the greater motivation to pursue optimal or near-optimal schedules in practice.

In summary, this limit study provides insights about several facets of GC design. First, it can tell us whether GC is essentially a solved problem (at least for some simpler models of collection) or whether further performance gains may be possible. Second, if we have a mechanism that gives some improvement, it can tell us how much of the potential improvement the mechanism is achieving. Third, it may be that improvement is possible for some programs and not others, so a limit study of a particular program can guide whether developing a program-specific GC mechanism might be worthwhile. The techniques we propose can guide future effort to decrease GC cost or heap space overhead, and may offer deeper insight into the nature and difficulty of the GC problem.

\section{TRACES AND GROUPS}

As described by Ricci et al. [10,11], ET traces consist of a sequence of event records, each recording an event associated with control (method calls, returns, etc.) or with memory management (object allocation, pointer updates, object death). Like its intellectual predecessor Merlin [7], ET computes precise death times for each object. Here we are interested in the subsequence of records pertaining to memory management, and these are entirely precise, lacking only a very small number of events that occur before ET can start or due to small deficiencies in the particular Java virtual machine's reporting of corner cases.

We are concerned here with three kinds of trace records: ones for object allocations, object deaths, and pointer updates. An object allocation record gives the object a unique ID (essentially a sequence number) and indicates its class and size, and for arrays, its number of elements. For our purposes, we develop object sizes using a uniform model of Java objects for a 64-bit platform. Specifically, scalar (non-array) objects have a two-word header (one word $=8$ bytes) plus their fields, packed as tightly as possible while obeying alignment constraints, rounded up to a multiple of 8 bytes. Each Java primitive type is aligned to a boundary equal to its size (char and short to 2 bytes, int and float to 4 bytes, long and double to 8 bytes). Arrays consist of a three-word header and their elements, also rounded up to a multiple of 8 bytes. This model could easily be adjusted to match that of any specific Java implementation-our algorithms do not depend in any way on the details of how each object's size is determined.

ET object death records indicate the object that died, giving its ID. These death times are precise with respect to the other events of interest, except that within a group of consecutive object deaths, their real death order cannot be determined. (In any case, one pointer update can cut off an arbitrarily large piece of the object graph, and all those objects die simultaneously. That is, even in the ideal, object deaths are only partially ordered.)

From the allocation and death records we can determine the volume of allocation in each group and the volume of live objects as of the beginning of the next group. This information is sufficient for computing our NG GC results.

For Gen GC we also need information about how objects refer to each other. ET's pointer update records enable us to model this, since they indicate the referring object, the target object, and the slot of the referring object that is being updated. It turns out that all we need to know is an object's outgoing references at the time it dies, which is easy to compute.

As previously mentioned, we aggregate object allocations into $256 \mathrm{~KB}$ groups. We start a new group when the next allocation would result in the current group being larger than the group size. Thus, objects whose size is $256 \mathrm{~KB}$ or more are allocated into a group by themselves. We allow collection to occur only just before the start of a new group, i.e., only at group boundaries. We feel this is reasonable since any actual policy cannot perform a complex computation at each object allocation. Computing the policy after every group is more realistic. 
Our motivation for using groups is the huge reduction in computation time and space needed to solve our models. It is important that the group boundaries be fixed, i.e., that they not depend on when a previous collection occurred, and so forth. We also find it convenient to have them fixed across heap configurations, so as to make results more comparable. Actual collectors also tend to restrict collection points; i.e., they do not introspect about whether to collect at every allocation. They will trigger collection when some region is filled or some threshold is reached. These dynamic triggers depend on when previous collections occurred, and in general their possible locations in the trace cannot be fixed in advance. To that extent our groupings do not exactly reflect reality. We present some results later for smaller group sizes to show how our aggregation into groups affects optimal schedule costs and the time to compute them. This allows some extrapolation as to optimal costs in the limit of allowing collection just before any allocation.

\section{MODELS OF GC}

We now present state machine formulations for both NG and Gen GC. ${ }^{4}$ We consider models with three primary components: a program $P$, a heap $H$, and a controller $C$. We assume that the model evolves in discrete time where the state variable $t$ represents the current timestep. On each timestep, the program $P$ first optionally performs some number of updates to the heap, and optionally causes some number of objects to die (become subject to reclamation by a future collection). It then does one of two things: (1) it allocates an object; or (2) it terminates. The optional actions cause state changes to the heap $H$, but are not visible to the controller $C$. If $P$ terminates, the machine stops. If $P$ allocates an object, the allocation request goes to $C$. $C$ optionally performs a GC, notifying $H$ to update its state, and then forwards the allocation request to $H$. $C$ may maintain state, and some parts of $H$ 's state may also be visible to $C$. This general structure applies to both NG and Gen GC. We note that this model assumes that the program $P$ cannot detect the reclamation of an unreachable object and change its behavior. Thus, we do not model features such as finalization or the special effects of Java's reference types.

\subsection{Modeling Non-Generational Collection}

From the point of view of the GC system, a running program can be described by a sequence of allocation requests from the program $P$ for objects, one per timestep $t$. We find it convenient to think of possible collections as occurring at the timesteps, i.e., at $0,1,2, \ldots, n$, if there are $n$ allocations in a run; and to think of the actual allocations and deaths as occurring between the timesteps. We map the actual birth and death times down to the previous step time, so if an object is allocated between steps $t$ and $t+1$, its birth time will be $t$, and so on. Thus, $t$ represents the number of objects allocated up to (before) time $t$ by $P$. An object $o_{t}$ has associated birth time $b\left(o_{t}\right)$, death time $d\left(o_{t}\right)$, and size in bytes $s\left(o_{t}\right)$. We let $\Omega$ be the set of objects that are allocated during a program run. If we number the objects in the order in which they are allocated, $b\left(o_{t}\right)=t$.

In the NG case, the heap itself can be described by a tuple of three state variables $\left(L_{t}, D_{t}, F_{t}\right)$ at each timestep $t$, as well as a static state variable $S$. $L_{t}$ represents the total size of live objects in the heap, $D_{t}$ represents the total size of dead objects in the heap, $F_{t}$ represents the total amount of free space in the heap, and $S$ represents the size of the heap. The heap maintains the invariant that $S=L_{t}+D_{t}+F_{t}$ at all times $t^{5}$

\footnotetext{
${ }^{4}$ Our previous work developed these as Markov Decision Processes, to map better to approximate optimal solution methods. Now that we have exactly optimal methods we can use a simpler state machine description.

${ }^{5}$ We consider only fixed size heaps. Some real collectors work that way, some do not. Here we show that, under the assumption of fixed size heaps, we can obtain an exact solution with a given polynomial cost. As we mention in Appendix B, our extension to multiple generations might form a basis for exact solutions in polynomial time when the heap size varies
} 
The controller implements a GC policy $\pi$. This policy is a function that maps the visible state of the rest of the system into the choice of an action $a$. The set of possible actions $\mathcal{A}$ is a property of the controller, $C$. In NG collection, the possible actions are collect and no-collect. However, some actions may not be available at a given timestep $t$. The set of available actions is denoted by $\mathcal{A}_{t} \subseteq \mathcal{A}$. For example, the choice not to collect on a given timestep $t$ is not valid if $s\left(o_{t}\right)$ (the size of the object to be allocated at timestep $t$ ) is larger than the remaining free heap space $F_{t}$. The visible state of the system at time $t$ is denoted $V_{t}$. The policy $\pi$ has access only to $V_{t}$, not the entire state. The contents of $V_{t}$ depend on the specific controller, but may contain the current timestep $t$, the current amount of free space in the heap $F_{t}$, the allocated space $A_{t}$ (defined as $L_{t}+D_{t}$ ), and a feature vector $X_{t}$ containing additional information about the state of the system obtained from suitably instrumenting the running program. (Although it is not strictly necessary, we find having both $A_{t}$ and $F_{t}$ to be convenient.) In this study, we take $X_{t}$ as being empty. Thus, $\pi$ maps visible state to legal actions: $\pi\left(V_{t}\right) \rightarrow \mathcal{A}_{t}$. We observe that since we include $t$ in $V_{t}$ and $\pi$ is defined in the context of a single program execution, $\pi$ can be structured as a lookup table based solely on $t$. We emphasize that this is not what a systems builder would call a policy! It is an oracle, not a mechanism. Thus, $\pi$ chooses a sequence of actions $a_{t}$, one for each timestep $t$. We call that sequence of actions a (GC) schedule.

The evolution of the state variables that define the system is driven by the sequence of allocation and death requests issued by the program $P$. The initial state of the heap prior to any allocations is $(0,0, S)$, indicating that all space is free. At each timestep $t$, the program requests the allocation of $s\left(o_{t}\right)$ bytes of storage for the object $o_{t}$ : alloc $\left(s\left(o_{t}\right)\right)$. As part of the same timestep, it announces the death of all objects that died since the last timestep (i.e., since the previous allocation) by issuing the set of notifications $\left\{\right.$ death $\left(o_{t^{\prime}}\right) \mid o_{t^{\prime}} \in \Omega$ and $\left.d\left(o_{t^{\prime}}\right)=t^{-}\right\}$. Here we use $t^{-}$to mean the timestep previous to $t .^{6}$ The controller $C$ receives the alloc $\left(s\left(o_{t}\right)\right)$ request and must fulfill the allocation by issuing an halloc $\left(s\left(o_{t}\right)\right)$ request to the heap $H$. However, before forwarding the request to the heap, the controller selects an action $a_{t} \in \mathcal{A}_{t}$ by applying the policy function $\pi(\cdot)$ to the visible state vector $V_{t}$.

To update the state of the heap, we first account for the objects announced as dead on timestep $t$. We let $\delta_{t}=\left\{t^{\prime} \mid o_{t^{\prime}} \in \Omega\right.$ and $\left.d\left(o_{t^{\prime}}\right)=t^{-}\right\}$be the set of (time points whose) objects that were announced as dead. We then let $\sigma_{t}=\sum_{t^{\prime} \in \delta_{t}} s\left(o_{t^{\prime}}\right)$ be the total number of bytes of all objects that were reported dead on timestep $t$. If the state of the heap on timestep $t$ is $\left(L_{t}, D_{t}, F_{t}\right)$, then after accounting for objects that were announced as dead, we have that the state of the heap is $\left(L_{t}-\sigma_{t}, D_{t}+\sigma_{t}, F_{t}\right)$

Now we take into account the action selected by the controller. We first note that the set of legal actions $\mathcal{A}_{t}$ is defined to be \{collect, no-collect\} if $F_{t} \geq s\left(o_{t}\right)$ and $\{$ collect\} otherwise. This forces a collection at timestep $t$ if there is insufficient space to allocate the object $o_{t}$. Once object $o_{t}$ has been allocated, the timestep is incremented. If the action $a_{t}$ selected by the controller is collect, the state of the heap is further updated to reclaim the space allocated to all dead objects. The updated state of the heap becomes $\left(L_{t}-\sigma_{t}, 0, F_{t}+D_{t}+\sigma_{t}\right)$. Following the allocation of object $o_{t}$, the state of the heap is $\left(L_{t}-\sigma_{t}+s\left(o_{t}\right), 0, F_{t}+D_{t}+\sigma_{t}-s\left(o_{t}\right)\right)$. If the action $a_{t}$ selected by the controller is no-collect, the updated state of the heap following the allocation of object $o_{t}$ becomes $\left(L_{t}-\sigma_{t}+s\left(o_{t}\right), D_{t}+\sigma_{t}, F_{t}-s\left(o_{t}\right)\right)$.

according to some known series of sizes over time, but the cost of solving that problem will be higher than for the fixed size case.

${ }^{6}$ This allows for consistency with similar numberings, such as defining $t$ in terms of bytes allocated so far, a common measure of time in formulating GC. 
Note that we assume collection does not require auxiliary space. This fits with implementations such as sliding compaction of the surviving objects in the heap.

The visible state consists of $t, A_{t}=L_{t}+D_{t}$, and $F_{t}$.

In summary, we thus have

$$
\begin{aligned}
L_{t+1} & \leftarrow \begin{cases}L_{t}-\sigma_{t}+s\left(o_{t}\right) & \text { collect } \\
L_{t}-\sigma_{t}+s\left(o_{t}\right) & \text { no-collect, }\end{cases} \\
D_{t+1} & \leftarrow \begin{cases}0 & \text { collect } \\
D_{t}+\sigma_{t} & \text { no-collect, }\end{cases} \\
F_{t+1} & \leftarrow \begin{cases}F_{t}+D_{t}+\sigma_{t}-s\left(o_{t}\right) & \text { collect } \\
F_{t}-s\left(o_{t}\right) & \text { no-collect. }\end{cases}
\end{aligned}
$$

It is now plain that the evolution of the state of the heap depends only on the three sequences $s\left(o_{t}\right), \sigma_{t}$, and $a_{t}$. Furthermore, we can see that the sequence of live size values $L_{t}$ is actually independent of $a_{t}$. As a corollary of the heap size invariant, we obtain the result that the free space $F_{t+1}$ obtained following a collection at time $t$ is always equal to $S-L_{t+1}$ since $D_{t+1}=0$. Since the sequence of values $L_{t}$ is independent of $a_{t}$, this means that the free space $F_{t+1}$ following a collection at time $t$ is independent of the sequence of prior actions. Finally, since $D_{t+1}=0$ following a collection, we obtain the result that the full state of the heap $\left(L_{t+1}, 0, S-L_{t+1}\right)$ following a collection is independent of the sequence of actions before time $t$. This fact will play an important role in designing algorithms for determining optimal GC schedules for the NG case. Note that our model assumes compaction, or at least that any fragmentation is already included in the size of the individual objects and does not change. Modeling fragmentation because of free-list allocation is beyond the scope of our dynamic program solution to the optimal GC schedule problem. ${ }^{7}$

We note that if $L_{t+1}>S$, the heap cannot hold the set of live objects. In that case, the machine terminates and indicates failure.

So far we have described how the state evolves, but have not yet indicated the cost associated with actions. We are interested in costs related to collection, not allocation or manipulation of the heap (which we do not model at all). We assume a tracing collector, and for NG define the cost of a collection to be $L_{t}$. That is, we define collection cost to be size of the live (reachable) objects. This holds to reasonable approximation for most tracing collectors, of both the mark-sweep and copying varieties. Many collectors handle large objects separately, especially ones that contain mostly nonpointer data. This can avoid work proportional to the size of those objects. Unfortunately, our dynamic program for finding optimal schedules becomes NP-complete for generational collection if we add such large object schemes. ${ }^{8}$

So far we have described allocation and collection on a per-object basis. How do groups enter into the model? As we said when we introduced groups, they restrict the points at which collection can occur. Therefore, the controller, $C$, must take groups into account. For NG, the change to the state machine rules is straightforward. For each group, we define the size of the group to be the sum of the sizes of the objects in the group, i.e., the space required in order to allocate the group contiguously. $C$ is presented with whole groups for allocation, and if the size of the next group is larger than $F_{t}$, then it must choose to collect, otherwise it may collect or not.

\footnotetext{
${ }^{7}$ Modeling collection under free-list allocation brings in the issue of which allocator one might model. In any case, the additional fragmentation and algorithmic concerns will probably make optimal solution an NP-complete problem.

${ }^{8}$ The reason is that a lower cost schedule that promotes a large object (at zero cost) reduces available old space for future collections. Our efficient solution for the generational case relies on the volume of the old space filled to increase as collection cost increases, so that lower cost implies less space used.
} 
While our evaluation uses heaps sizes that are an integral multiple of the group size, because groups vary in size, even in the absence of single-object groups, the number of groups that can fit in the heap at once can vary. However, $g$ groups of space will contain fewer than $2 \cdot g$ groups. This is because any two adjacent groups must contain more than $G$ bytes (where $G$ is the group size)-otherwise they would have been combined into one group.

\subsection{Modeling Generational Collection}

The Gen case requires several modifications to the previous model. First, the model for the heap is extended to include separate young and old sections with sizes $S_{Y}$ and $S_{O} .{ }^{9}$ The set of actions that the controller can perform is augmented to allow collection of only the young section of the heap, or collection of the full heap. The action set is thus $\mathcal{A}=$ yyoung-collect, full-collect, no-collect $\}$. As in the NG case, only a subset of actions $\mathcal{A}_{t} \subseteq \mathcal{A}$ is available at each timestep, but the calculation of $\mathcal{A}_{t}$ is now slightly more complicated since we must ensure that neither the young nor the old space overflow.

Note that allocations, except for very large objects, are into young space, with collections promoting certain young space objects to old space. ${ }^{10}$ An object larger than young space will be allocated directly to old space. We force objects to be considered for promotion in age order (oldest first). Therefore, allocating a very large object requires young space to be empty, and thus may force a collection first.

The heap is modeled by the tuple $\left(L_{t}^{Y}, L_{t}^{O}, D_{t}^{Y}, D_{t}^{O}, F_{t}^{Y}, F_{t}^{O}, B_{t}, T_{t}^{Y}, T_{t}^{O}\right)$. The superscript $Y$ indicates state variables related to the young section of the heap and $O$ those related to the old section. The $L$ variables represent the live size of a section, the $D$ variables represent the dead size, and the $F$ variables the free space. $B_{t}$ is the total amount of "baggage" in the young section of the heap. The baggage is the total number of bytes of dead objects that will be promoted to the old section of the heap if only the young section of the heap is collected. The variables $T_{t}^{Y}$ and $T_{t}^{O}$ indicate the timestep on which the young space and the old space were last collected (note that a young collection updates $T_{t}^{Y}$ only, while a full collection updates both $T_{t}^{Y}$ and $T_{t}^{O}$ ). This expanded model includes two heap invariants, one for each section of the heap: $L_{t}^{O}+D_{t}^{O}+F_{t}^{O}=S_{O}$ and $L_{t}^{Y}+D_{t}^{Y}+F_{t}^{Y}+B_{t}=S_{Y}$.

The dynamics of full collections work identically to the single-heap case. However, the dynamics of a young collection are more nuanced. When a young collection is performed, all live objects in the young section of the heap are copied to the old section of the heap. However, dead objects in the young generation that are pointed to by objects in the old generation appear still to be live during a young collection, and thus are also copied to the old section of the heap. To support this reasoning accurately, the model of objects is expanded to include a pre-birth time, as presented in Section 4 , notated $p\left(o_{t}\right)$.

The initial conditions for the heap are $L_{t}^{Y}=0, L_{t}^{O}=0, D_{t}^{Y}=0, D_{t}^{O}=0, F_{t}^{Y}=S_{Y}, F_{t}^{O}=S_{O}$, and $B_{t}=0$. We need several auxiliary sequences to keep track of the objects that die at each timestep. First, we let $\delta_{t}^{O}=\left\{t^{\prime} \mid o_{t^{\prime}} \in \Omega, d\left(o_{t^{\prime}}\right)=t^{-}, b\left(o_{t^{\prime}}\right)<T_{t}^{Y}\right\}$. This set contains the indices of all objects reported dead at time $t$, and that were born before the last young collection. This means these objects were promoted to the old space. Their total size is given by $\sigma_{t}^{O}=\sum_{t^{\prime} \in \delta_{t}^{O}} s\left(o_{t^{\prime}}\right)$. Next, we let $\delta_{t}^{B}=\left\{t^{\prime} \mid o_{t^{\prime}} \in \Omega, s\left(o_{t^{\prime}}\right)=t, b\left(o_{t^{\prime}}\right) \geq T_{t}^{Y}, p\left(o_{t^{\prime}}\right)<T_{t}^{Y}\right\}$. This set contains the indices of all objects

\footnotetext{
${ }^{9}$ We realize that in this section $Y$ and $O$ are mostly used as superscripts. We apply them to $S$ as subscripts so that later we can write powers of them readably, e.g., $S_{Y}^{1.2}$.

${ }^{10} \mathrm{We}$ assume all reachable young space objects are promoted, i.e., that objects do not have to survive more than one collection to be promoted, and so forth. Such schemes would entail a more complex analysis. The extension in Appendix B might be adapted to handle them.
} 
there were reported dead at time $t$, and were born after the last young collection, but have a pre-birth time before the last young collection. These objects are pointed to by objects in the old space at the time they die, and thus contribute to the baggage in the young generation. Their total size is $\sigma_{t}^{B}=\sum_{t^{\prime} \in \delta_{t}^{B}} s\left(o_{t^{\prime}}\right)$. Finally, let $\delta_{t}^{Y}=\left\{t^{\prime} \mid o_{t^{\prime}} \in \Omega, d\left(o_{t^{\prime}}\right)=t^{-}, b\left(o_{t^{\prime}}\right) \geq T_{t}^{Y}, p\left(o_{t^{\prime}}\right) \geq T_{t}^{Y}\right\}$. This set contains the indices of all objects reported dead at time $t$, that were born after the last young collection, and have a pre-birth time after the last young collection. These objects are correctly identified as being dead within the young space. Their total size is $\sigma_{t}^{Y}=\sum_{t^{\prime} \in \delta_{t}^{Y}} s^{s}\left(o_{t^{\prime}}\right)$. We further define $\sigma_{t}=\sigma_{t}^{O}+\sigma_{t}^{B}+\sigma_{t}^{Y}$.

Our cost and space consumption model assumes that young collections copy retained objects from young space to old space, and that full collections compact old space without using auxiliary space. Again, this fits with old space collection implementations such as sliding compaction.

The visible state consists of $t, A_{t}^{Y}=L_{t}^{Y}+D_{t}^{Y}+B_{t}^{Y}, A_{t}^{O}=L_{t}^{O}+D_{t}^{O}, F_{t}^{Y}, F_{t}^{O}, T_{t}^{Y}$, and $T_{t}^{O}$.

We can now specify the full dynamics of generational collection:

$$
\begin{aligned}
& L_{t+1}^{O} \leftarrow \begin{cases}L_{t}^{O}+L_{t}^{Y}-\sigma_{t}^{O} & \text { full/young-collect } \\
L_{t}^{O}-\sigma_{t}^{O} & \text { no-collect, }\end{cases} \\
& D_{t+1}^{O} \leftarrow \begin{cases}0 & \text { full-collect } \\
D_{t}^{O}+B_{t}+\sigma_{t}^{O} & \text { young-collect } \\
D_{t}^{O}+\sigma_{t}^{O} & \text { no-collect, }\end{cases} \\
& F_{t+1}^{O} \leftarrow \begin{cases}F_{t}^{O}-L_{t}^{Y}+D_{t}^{O}+\sigma_{t}^{O} & \text { full-collect } \\
F_{t}^{O}-L_{t}^{Y}-B_{t} & \text { young-collect } \\
F_{t}^{O} & \text { no-collect, }\end{cases} \\
& L_{t+1}^{Y} \leftarrow \begin{cases}s\left(o_{t}\right) & \text { full/young-collect } \\
L_{t}^{Y}-\sigma_{t}^{Y}-\sigma_{t}^{B}+s\left(o_{t}\right) & \text { no-collect, }\end{cases} \\
& D_{t+1}^{Y} \leftarrow \begin{cases}0 & \text { full/young-collect } \\
D_{t}+\sigma_{t}^{Y} & \text { no-collect, }\end{cases} \\
& F_{t+1}^{Y} \leftarrow \begin{cases}S_{Y}-s\left(o_{t}\right) \\
F_{t}^{Y}-s\left(o_{t}\right)\end{cases} \\
& B_{t+1} \leftarrow \begin{cases}0 & \text { full/young-collect } \\
B_{t}+\sigma_{t}^{B} & \text { no-collect, }\end{cases} \\
& T_{t+1}^{Y} \leftarrow \begin{cases}t & \text { full/young-collect } \\
T_{t}^{Y} & \text { no-collect, }\end{cases} \\
& T_{t+1}^{O} \leftarrow \begin{cases}t & \text { full/young-collect } \\
T_{t}^{O} & \text { no-collect, }\end{cases}
\end{aligned}
$$

Heap overflow: We have found it convenient to allow some actions to fail when others are successful. In fact, we can model no-collect as failing when there is not enough free space. In the Gen case, however, young-collect can lead to failure in some cases where full-collect does not. This occurs when a young collection would promote objects that would overflow the old space, but the total live volume at this point is still less than $S_{O}$. A real collector would prefer to avoid failure if some action leads to success, though this may involve either (a) being conservative and doing a full-collect in case a young-collect might lead to overflow, or (b) designing the collector so that it can optimistically start a young-collect but switch to full collection if the young-collect would fail. We compute optimal cost assuming no extra overhead from such a mechanism. This is equivalent to having the optimistic mechanism available but a perfect predictor as to whether to perform a full-collect instead of the optimistic young-collect with fallback. 
Cost Model: As for NG, we define cost in terms of the volume of objects traced by a tracing collector. Therefore, the cost of a full-collect is simply $L_{t}^{Y}+L_{t}^{O}$, the cost of a young-collect is $L_{t}^{Y}+B_{t}$, and the cost of no collection is 0 .

The rules above do not handle the case of a (single-object) group of size $S_{Y}$ or more. In that case, if the young space is not empty (i.e., if $F^{Y}<S_{Y}$ ), then a collection is required. After the collection, the object is allocated directly to old space, adjusting $L_{t+1}^{O}$, and so forth, appropriately.

Group Allocation: As for NG, $C$ is presented with whole groups and must make action decisions based on the size of the entire group. As described in Section 4, we can determine baggage size, and so forth, using summary pre-birth, birth, and death times for sets of objects in a group that will all be treated the same (what we will call cohorts). We now turn to this refinement.

\section{GENERATIONAL GC COST COMPUTATION}

There are two aspects of our approach to efficient computation of the cost of Gen GC that we now treat in detail. The first concerns a method to precompute information related to the object graph that (a) simplifies computing the cost of a Gen GC schedule and (b) reduces the cost of our dynamic program. In particular, we introduce what we call the pre-birth time of an object. An object's prebirth time, along with its birth and death time, determines when that object will be promoted by a young GC, without needing to build or traverse the object graph. The second aspect is the notion of cohorts of objects, where all objects in a given cohort will have the same behavior under Gen GC, and how to compute those cohorts. Cohorts associate objects based on groups and thus also reduce the cost of computing GC costs in both space and time. If one were computing collection cost for just one schedule of collections, then these techniques would not improve performance. However, to find optimal schedules we evaluate the cost of many partial and full schedules, and this pre-computation is therefore of significant benefit.

\subsection{Pre-Birth Times}

Given the object birth and death time information available from our traces, it is simple to compute the set of live objects at a full GC. Starting from sorted birth and death records, one can process a trace in order and track the live size at all time steps with constant work per trace record. As will be seen, our cost model for full GC is based on the volume of live objects, so it is therefore cheap to compute the cost of a full GC at any timestep.

Unfortunately, the situation is not so straightforward with young GCs. Of course, a live object in young space will be promoted to old space, and a live object in old space will be retained. However, dead objects in old space will also be retained, and if they refer to dead objects in young space, those objects will be promoted. We used the term baggage for such promoted dead objects, and they increase the cost of a young GC. They also take up room in the old space, possibly forcing a full GC sooner than would otherwise be necessary.

Consider an object $x$ with birth time $b(x)$ and death time $d(x)$. Our unit of time is the number of objects allocated so far, so $b(x)$ is the number of objects allocated before $x$, while $d(x)$ is the number of objects allocated before the interval in which $x$ dies. Necessarily, $d(x) \geq b(x)$, but observe that it is possible for an object to be born and then to die before the next collection, in which case $b(x)=d(x)$. (Supposing $b(x)=t$, then the birth and death both occur in the interval $(t, t+1)$, so given how we define $d(x)$, it also equals $t$.) It may be helpful to think of $b(x)$ and $d(x)$ as occurring infinitesimally after their actual values. This clarifies that a collect "at" $b(x)$ or $d(x)$ is actually before that birth or death.

Now, given a dead object $x$, consider all objects that died at or before $d(x)$, and in particular consider their pointers from one to another. We observe that this part of the object graph will 


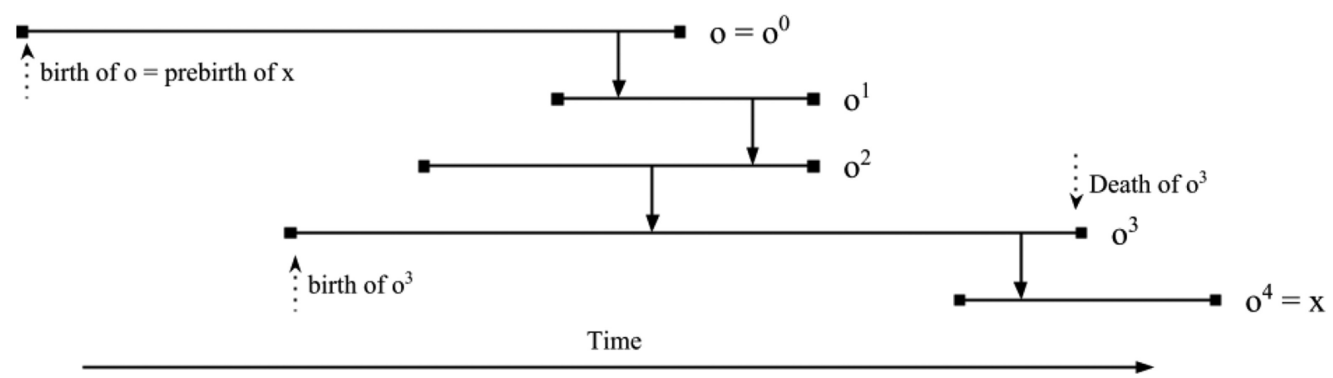

Fig. 1. Sample of pre-birth time.

never change after $x$ 's death (the program $P$ cannot access dead objects and so cannot change them). Now consider the subset of these dead objects from which there are paths to $x$. We call these the predecessors of $x$ in the dead object graph. ${ }^{11}$ Let $o$ be the predecessor of $x$ whose birth time is the earliest.

Claim: A young collection after $d(x)$ will promote $x$ if and only if there is a collection (young or full) after $b(o)$ (the birth time of $x$ 's oldest predecessor) and before $b(x)$, and no collection between $b(x)$ and $d(x)$. Proof: First suppose there is no collection between $b(o)$ and $d(x)$. Then collection after $d(x)$ will reclaim, not promote, $x$ and all its predecessors in the dead object graph. To see this, we note that if $y$ is dead and refers to $x$, then $d(x) \geq d(y)$, since if $d(y)>d(x)$, the lifetime of $x$ would be extended. In other words, object $x$ cannot die before another object $y$ that refers to $x$ at the time $y$ dies. This property holds inductively. Thus, all unreclaimed predecessors of $x$ in the (dead) object graph were born at or after $b(o)$ and died at or before $d(x)$. They must all lie in young space and will be reclaimed by any collection that occurs after $d(x)$. None of them are pointed to by any object in old space.

Now we tackle the converse. Suppose there is a collection between $b(o)$ and $b(x)$ and no collection while $x$ is live. We argue that there is some object $y$ in old space that, via a chain of one or more references, refers to $x$ and forces $x$ to be promoted if there is a young collection after $d(x)$. Consider a chain of objects $o=o^{0}, o^{1}, \ldots, o^{n}, o^{n+1}=x$ where each of these objects refers to the next in the dead object graph. Since $o^{i}$ refers to $o^{i+1}$ (for $\left.i=0, \ldots, n\right), o^{i}$ and $o^{i+1}$ have overlapping lifetimes (the mutator made $o^{i}$ refer to $o^{i+1}$, so they were both live at that moment). Therefore, the chain of objects together spans lifetimes covering the range $b(o)$ through $d(x)$. Therefore, for any time $t^{\prime}$ such that $b(o) \leq t^{\prime} \leq b(x)$, at least one object in the chain will be live at time $t^{\prime}$ and be promoted. This applies specifically to the latest such $t^{\prime}$. Note that our argument depends on restricting collection to occur in age order. That is, if $b(x)<b(y)$, then $y$ cannot be collected before $x$. Most collectors work this way because of faith in the generational hypothesis [6], though collecting in this order is not a logical necessity.

A minor part of the converse is that collection between $b(x)$ and $d(x)$ will promote $x$, but this is obvious since $x$ is live in that interval.

Figure 1 illustrates a sample pre-birth situation. Each horizontal line corresponds to the lifetime of one object, its birth time marked by the left end of the line and its death time by the right end, time passing from left to right. Each solid vertical arrow indicates a pointer from the object above to the object below, such that the pointer still exists when the upper object dies, and the pointer is created at the time where the arrow falls on the time line. Notice that the death times, working

\footnotetext{
${ }^{11}$ We emphasize that since $x$ is dead, there can be no (directed) path to $x$ from a live object. This is inherent in the definition of live vs. dead.
} 
from $o^{0}$ to $o^{4}$, are non-decreasing. This is because the continued reachability of the upper object makes the lower object reachable at least until the upper one dies. However, the birth times can bear any relative relationship, with the exception that the birth of $o^{0}$ is necessarily earliest (because of how we defined $o^{0}$ ). Also, a vertical arrow must fall during the lifetime of both the upper object and the lower one. From this diagram one can see that for any time between the birth of $o^{0}$ and the death of $o^{4}$, either $o^{4}$ is live, or one of its predecessors is, in this fragment of a dead object graph.

In our argument using the dead object graph, we considered the oldest dead object $o$ from which the target object $x$ is reachable. We call $b(o)$ the pre-birth time of $x$, written $p(x)$. The end result of this argument is that if the previous collection occurred after $p(x)$, then a young collection with $x$ in young space will cause $x$ to be promoted, whether or not $x$ is live. The point is that a precomputation over the dead object graph can provide the additional per-object datum, $p(x)$, sufficient for calculating young object promotions. We do not need to build the object graph and traverse it, simulating GC directly, in order to evaluate the cost of a GC schedule. We can compute the derived property $p(x)$ once, and use it, with $b(x)$ and $d(x)$, to determine what will happen to $x$ in any sequence of collections.

Furthermore, sets of objects can be sorted in terms of these times to make simulation of the effects of collection very efficient. Here is how such an efficient per-object simulation can proceed. We keep track of the time $c$ when the most recent collection occurred. When a new object $x$ is allocated, if $p(x)<c$, then we add $x$ to a baggage set, which is sorted by death time. If $p(x) \geq c$, we add it to a non-baggage set, also sorted by death time. When a young collection occurs at time $t$, the baggage set objects are promoted, as well as non-baggage set objects whose death time is greater than $t$.

The old space object set is also maintained in death time order. A full GC discards objects in all three sets (young baggage, young non-baggage, and old) whose death time is no more than $t$ and puts the remaining objects in the old space set. Each object is handled at most three times (allocation, promotion, death), with each operation's cost logarithmic in the number of objects that can fit in the heap, i.e., logarithmic in heap size. This gives a total simulation cost of $O(n \log H)$ where $n$ is the number of objects and $H$ is the heap size. Simulating actual GC directly requires building and traversing object graphs with worst-case cost $O(n \cdot H)$. So, for repeated simulations our precomputation can save a lot.

Here is how to compute pre-birth times efficiently. When processing an ET trace, maintain a model of the objects allocated in the heap. Associate with each object its pre-birth, birth, and death times. When processing an allocation event record, we allocate the new object in the model of the heap, assign its birth time according to the number of objects allocated so far, and set its initial pre-birth time to its birth time. When processing a pointer update event, we update the heap model to reflect the pointer update. When processing a death record, we propagate the pre-birth time of the dying object to all objects to which the dying object refers. By "propagate" we mean to set the target object's pre-birth time to the minimum of its current pre-birth time and the pre-birth time being propagated, and if that reduces the target object's pre-birth time, propagate recursively to the objects to which the target refers. Since a number of objects may die at the same time, it is necessary to do all these propagations before removing any of the dying objects from the heap, since another object's death might cause pre-birth values to propagate through an object dying at the same time. Once all the deaths at a given time have been processed, the newly dead objects have stable pre-birth times, and their three times (pre-birth, birth, and death) can be recorded and the objects removed from the heap model. This will result in a trace of object information in death order. This trace will need to be sorted into birth order to be suitable for the heap simulation previously described. 


\subsection{Group-Based Collection: Cohorts}

As already revealed, a cohort is a set of objects where all the objects will behave the same under group-oriented Gen GC. However, we need to unpack what we mean by "behave the same."

Since we restrict collection to occur only at group boundaries, we can aggregate objects according to groups. We can map object pre-birth, birth, and death times to the group within whose allocation times those object times fall. (Here it is helpful to recall that we consider births (and pre-births) to occur infinitesimally after the integer time values we record for them, and deaths to occur infinitesimally before the value we record.) We call these groups the pre-birth, birth, and death groups of the object. A cohort is a set of objects that share the same pre-birth, birth, and death groups. Given our previous result on pre-birth times, and the restriction that collection occurs only on group boundaries, we can compute collection costs from our knowledge of the size of each cohort, avoiding the need to handle individual objects. This can significantly speed our cost calculations. In our argument concerning pre-birth times, we explained how GC behavior could be simulated handling each object at most three times. This same strategy applies to cohorts, giving algorithms that handle each cohort only three times.

In the limit of small group sizes, each group will contain one object and cohorts become the same as objects, but for larger group sizes there can be significant savings. In principle, if there are $g$ groups, there could be up to $O\left(g^{3}\right)$ cohorts. However, the number of cohorts whose birth group is a given group is limited by the maximum number of objects that fit in a group. If the group size is $G$ bytes and the minimum object size is $m$ bytes, then at most $G / m$ cohorts can have the same birth group. Thus, assuming $g$ groups, there are at most $g \cdot G / m$ cohorts. Put another way, the number of cohorts is at most a constant factor times the number of groups, and thus in the limit of long traces the number of cohorts grows at most proportionally with the length of the trace. Since $g$ is roughly inversely proportional to $G$, the number of cohorts for a trace of the same length will not change much as we vary $G$, except at the extremes of relatively small or large values for $G$ (relative to the distribution of object sizes in the trace).

It is clear that restricting collection to occur only at group boundaries means we do not necessarily find the very lowest cost GC schedules compared with allowing collection just before any allocation. While we do not do so here, we believe that it is possible to develop bounds on the gap between optimal collection without this restriction and with the group boundary restriction. For example, for NG GC, the error is less than $G$ per collection, and can be tightened further by considering the minimum live size within each group. It is rather more complex for Gen GC, so we leave detailed investigation to future work. Suffice it to say that it appears that getting an exact result in the unrestricted case appears infeasible for traces large enough to be interesting.

While collecting only at group boundaries is pragmatic, it is also realistic in that many collectors work on a block basis. A thread acquires one block at a time from a global pool, and then allocates sequentially (so-called "bump pointer" allocation) within the block. Making collection decisions at group boundaries is thus natural, though admittedly not all collectors work this way.

How exactly does the group size $G$ affect the problem size and solution cost? Intuitively, halving the group size approximately doubles the number of groups. However, if a group is already restricted to contain one object, e.g., because the object is larger than the group size, reducing the group size will not split the group. As we shrink the group size, this will happen more often. We show the relationship empirically in Section 7. We noted above that an upper bound on the number of cohorts may not be strongly affected when changing $G$, unless we are near the extremes of object sizes for the trace. As with the number of groups, we present results on the number of cohorts in Section 6. 
In sum, our precomputed pre-birth times allow us to reduce repeated GC simulation cost from an $O(H)$ factor to an $O(\log H)$ one, ${ }^{12}$ where $H$ is the heap size. That is, determining baggage, and so forth, at each possible collection point, in the absence of our pre-birth information, requires tracing the heap (simulating a young collection), whose worst-case cost is $O(H)$, i.e., one may have to examine the entire heap. However, given our pre-birth information, we simply access that information from sorted data structures whose size is the current occupancy of the heap, giving $\operatorname{cost} O(\log H)$. The group boundary restriction further reduces costs by a factor of about $(G / s)^{2}$, where $G$ is the group size and $s$ is the average object size.

\section{DYNAMIC PROGRAMS FOR FINDING OPTIMAL SCHEDULES}

Before we explain our dynamic programs, we offer an overview and present some preliminary definitions. Both of our dynamic programs rely on simple preliminary computation of the amount of data allocation in each group and the live size at group boundaries. For NG collection, the cost of collection is simply the live size $L[t]$ when the collector is triggered at time $t$, and collections can only be so far apart: the live size at the previous collection, plus the amount allocated since, must fit in the given heap size $S$. It is straightforward to develop a dynamic program that gives the optimal cost $F[t]$, and an optimal schedule, when collection occurs at a given time $t$. The optimal solution across the whole run is then determined by the optimal cost when there is a collection at the end. (At that point the live size, and thus the cost, is 0 .)

For the Gen case, we develop the dynamic program in two large stages. The first stage considers only young collections. It addresses the constraint of young space size, but ignores the size of old space. It develops arrays $y[i, j], Y[i, j]$, and $\bar{Y}[i, j]$. The second stage introduces full collections and considers their optimal placement given the model of optimal young collection placement. It develops arrays $f[i, j]$ and $F[t]$. This two-stage development ignores the consideration of objects that meet or exceed the size of young space, so we add a refinement that deals with that. It develops an array $B[t]$ (for "big").

Here are the preliminary definitions. First, indices for indexed values in our dynamic programs are group numbers. The group size (sometimes called the block size) is notated $G$. We use $n$ as the number of groups in a given trace (for a given $G$ ). Grouping defines $n+1$ positions in the trace (on either side of the $n$ groups, like fence posts and a fence), which we number 0 through $n$. If $A$ is the total number of bytes allocated, then $n<2 A / G$. (The factor of 2 comes from the worst-case grouping where each object is just over $\frac{1}{2} G$ in size.)

Every object $x$ has a pre-birth time $p(x)$, a birth time $b(x)$, and death time $d(x)$. We use $V[\ldots]$ to denote subscripting of the various arrays of our dynamic programs. Necessarily, $p(x) \leq b(x) \leq$ $d(x)$. Here time is the number of bytes allocated so far in the trace. It is helpful to think of the given times as picking out instants, and the events as occurring between these instants. So, if for some object $x$ we have $p(x)=b(x)=d(x)=t$, we know that the object was pre-born, born, and died at some times between the instants picked out at $t$ and $t+1$. This helps clarify what happens when we consider a GC to happen "at" time $t$-for a full collection, objects $x$ with $b(x)<t \leq d(x)$ are live and will be retained. Others are either dead $(d(x)<t)$ and will be reclaimed or have not yet been allocated $(t \leq b(x))$.

The $p, b$, and $d$ of each object are mapped to the group they fall within, the groups being numbered 0 through $n-1$. Objects with the same mapped $(p, b, d)$ triple exhibit the same collection behavior and form a cohort. We define $c[p, b, d]$ to be the size of the cohort with pre-birth group

\footnotetext{
${ }^{12}$ Actually, full simulation could be even worse, and is proportional to the length of the Elephant Tracks traces. These traces contain pointer update records, and since any given pointer can be updated arbitrarily many times, the full trace need not be of length $O(H)$.
} 
$p$, birth group $b$, and death group $d$, i.e., the sum of the sizes of the objects in the cohort. Here $p \leq b \leq d$. The values of $c[\cdot, \cdot, \cdot]$ completely capture the behavior of the trace for the given group size, and form the basis of the optimization problem to be solved.

Given $c[\cdot, \cdot, \cdot]$ it is easy to define the live size, or volume of data that is live at a given point:

$$
L[t]=\sum_{b<t \leq d} c[p, b, d] .
$$

No data are live before the trace begins and after it ends, so we have $L[0]=L[n]=0$.

Similarly, we define $A[i, j]$ to be the volume of data allocated between $i$ and $j$. It is calculated as

$$
A[i, j]=\sum_{i \leq b<j} c[p, b, d] .
$$

\subsection{Dynamic Program for Optimal NG Schedules}

While our goal is solving the harder problem of Gen schedules, for completeness we offer a dynamic program for NG. This will turn out to include some observations that are helpful in improving the asymptotic performance for the Gen case as well.

Recall that $S$ denotes the size of the heap. We define $f[i, j]$ to be the cost of collecting at time $j$ given that the previous collection was at time $i$, with no collections allowed in the middle. If allocation between $i$ and $j$ would overflow the heap, we define $f[i, j]$ to be $\infty$, as expressed in this equation:

$$
f[i, j]= \begin{cases}L[j] & \text { if } L[i]+A[i, j] \leq S \\ \infty & \text { otherwise. }\end{cases}
$$

The point of this definition is to capture the effect of bounded heap size.

To compute the optimal overall schedule cost, we define $F[j]$ to be the minimum cost of a valid schedule that begins at the start of the trace and ends with a collection at $j$ :

$$
F[j]=\min \left\{\begin{array}{l}
f[0, j], \\
\min _{0<k<j} F[k]+f[k, j] .
\end{array}\right.
$$

The first term covers the case where the first collection in the schedule is at $j$, while the second one covers the case of at least one prior collection, where the last of those collections is at $k$. Notice that there can be at most $2 S / G$ groups before the sum of the sizes of the groups exceeds $S$. Therefore, when $(k-j)>2 S / G, f[k, j]$ will be $\infty$. That is, $f[k, j]$ will be finite for only a band whose width is bounded by a constant. This means that the min of the equation is over only a constant number of terms.

Finally, the minimum schedule cost is given by $F[n]$, since a collection at $n$ after the end of the trace has no cost. If we precompute and save $A[0, i]$, which is a simple prefix sum, we can compute (rather than store) $A[i, j]$ for the cases we need as $A[0, j]-A[0, i]$. Thus, computing the needed elements of $f[i, j]$ will require a total time that is $O(n)$. Given those values, computing the $F[j]$ table entries takes an additional $O(n)$. The overall cost is dominated asymptotically by the cost to sort cohort information in birth order and death order, $O(n \log n)$. The space required is $O(n)$.

We also observe that $L[\cdot]$ and $A[\cdot, \cdot]$ do not depend on pre-birth times, so this dynamic program can use a simpler form of cohorts based only on birth and death times, and need not perform the possibly expensive computation of pre-birth times at all.

\subsection{Dynamic Program for Optimal Gen Schedules}

The algorithm proceeds by filling in a series of dynamic programming tables-several more than in the NG case-each of which builds on the last. We now describe these tables in turn. 
First, consider the cohorts that are promoted ${ }^{13}$ during a young collection. They fall into two categories. The first is the live objects that have not yet been promoted, i.e., objects that were born after the previous collection but have not yet died. The second is baggage, the objects that were pre-born during the previous collection and have since died. All together this implies that, in order to calculate the cost of a young collection and the volume of data that it promotes, we need only one piece of information in addition to the properties of the cohorts themselves: the timestep of the previous collection. This allows us to define $y[i, j]$, which is the cost of a young collection at timestep $j$, given that the previous collection was at step $i$. (Necessarily $i<j$.) For convenience, we adopt the convention that $y[i, j]$ is infinite if the volume of data allocated between $i$ and $j$ is larger than our young space size, $S_{Y}$. Formally, we calculate $y[i, j]$ as

$$
y[i, j]=\left\{\begin{array}{lll} 
& \sum_{i \leq b<j \wedge j \leq d} \quad c[p, b, d] \text { (live) } & \\
+ & \sum_{p<i \wedge i \leq b \wedge d<j} c[p, b, d] \text { (baggage), } & \text { if } A[i, j] \leq S_{Y} \\
\infty & & \text { otherwise }
\end{array}\right.
$$

Again, $y[i, j]$ is the cost of a young collection at time $j$ assuming the previous collection (young or full) was at $i$, i.e., there is no collection in between. (This intentionally does not include the cost of the collection at $i$, only that of the collection at $j$.)

Next, we widen our focus to schedules of collections. Let $Y[i, j]$ be the minimum cost of any schedule that (a) begins with a collection (young or full) at $i$, (b) ends with a young collection at $j$, and (c) has zero or more young collections in between. Note that because the cost is the volume of data promoted, the same schedule minimizes bytes traced and bytes promoted. This is important in determining impact on occupancy in the old space. As with $y[i, j], Y[i, j]$ includes the cost of collecting at $j$ but not the cost of collecting at $i$. Collecting a schedule of zero length has no cost, so we begin with $Y[i, i]=0$. Then, we can calculate $Y[i, j]$ recursively:

$$
Y[i, j]=\min \left\{\begin{array}{l}
y[i, j], \\
\min _{i<k<j} Y[i, k]+y[k, j] .
\end{array}\right.
$$

There are two possible cases. First, there may be no young collections between $i$ and $j$, giving cost $y[i, j]$. (Recall that if that is not feasible, $y[i, j]=\infty$.) Second, there could be one or more young collections between $i$ and $j$. In this case, let $k$ be the location of the last such collection. Then, we can split the entire range into two smaller ranges, one from $i$ to $k$ and another from $k$ to $j$. We have met the constraint that there is a collection at the end of each range, so the total cost of the schedule from $i$ to $j$ becomes the sum of the costs of the shorter segments: $Y[i, k]+y[k, j]$. Of course, there may be many possible ways to split the range into two segments. We choose the one that has the lowest cost. It is important in our later analysis that the second component of this sum is $y$ as opposed to $Y$.

In practice, we calculate $Y$ in a dynamic programming table. The value of a cell for a certain range depends only on the values for shorter ranges. So we initialize values along the diagonal to be 0 , and can then work across each row (more generally, upward from the diagonal).

Next, it is useful to relax the restriction that there must be a young collection exactly at the end of a certain range. This yields $\bar{Y}[i, j]$, which represents the minimum cost of a schedule from $i$ to $j$ that allows only young collections. Intuitively, we begin at each cell of $Y[i, j]$ and look backward for the minimum cost schedule that ends within one young space size of $j$. The calculation of $\bar{Y}$ is straightforward:

$$
\bar{Y}[i, j]=\min _{\left\{j^{\prime} \mid i \leq j^{\prime} \leq j \wedge A\left[j^{\prime}, j\right] \leq S_{Y}\right\}} Y\left[i, j^{\prime}\right] .
$$

\footnotetext{
${ }^{13}$ Observe that our cost model views promotion as essentially implying copying, so we tend to use the terms synonymously.
} 
Finally, we calculate the costs of schedules that include full collections. We define $f[i, j]$ analogously to $y[i, j]$ : it is the cost of a schedule that begins with a full collection at $i$ and ends with a full collection at $j$, but has no full collections between $i$ and $j$. It does, however, include the costs of any young collections between $i$ and $j$. As with $y$, and so forth, it includes the cost of the collection at $j$, but not of the one at $i$. We further ensure that the amount of data promoted fits into the old space. That constraint has to do with the space in use at $i$, namely, $L[i]$, plus the volume promoted during $i$ to $j$.

$$
f[i, j]= \begin{cases}\bar{Y}[i, j]+L[j] & \text { if } L[i]+\bar{Y}[i, j] \leq S_{O} \\ \infty & \text { otherwise. }\end{cases}
$$

At last we are ready to calculate the optimal schedules with arbitrarily placed young and old collections. We let $F[j]$ be the minimum cost of a schedule that begins at 0 and ends with a full collection at $j$. This is analogous to the first row of $Y[i, j]$. It would be straightforward to extend $F$ to be a two-dimensional table, but the extra data are not needed for our purposes and would increase the cost of solving the problem. $F[0]=0$, since there is no cost to collect a schedule of length 0 . The calculation proceeds almost identically to $Y[0, \cdot]$ :

$$
F[j]=\min \left\{\begin{array}{l}
f[0, j], \\
\min _{0<k<j} F[k]+f[k, j] .
\end{array}\right.
$$

As before, we consider the cases where there are no full collections between 0 and $j$, and those where there are one or more. We take the minimum cost of all the various options. We again calculate these values using a dynamic programming table.

After the end of the trace, all objects have died, so there is no cost to collecting at $n$. This means that the minimum cost of a schedule for an entire trace can be read from the table: it is $F[n]$.

There is one additional complication we have glossed over. Objects larger than the young space are allocated directly into the old space, provided that the young space is empty at the time. Handling this condition requires some extra bookkeeping. We let $B[i]$ be the size of the group allocated at $i$, if it is at least as large as the young space size, and 0 otherwise. That is,

$$
B[i]= \begin{cases}A[i, i+1], & \text { if } A[i, i+1] \geq S_{Y} \\ 0 & \text { otherwise. }\end{cases}
$$

Then, we make the following modifications. First, the calculation of $y$ becomes

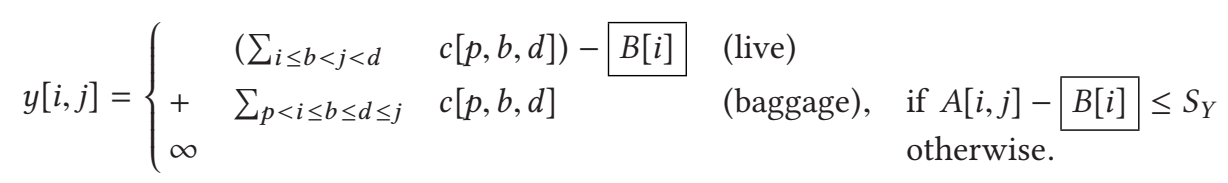

This ensures that allocation directly into the old space is allowed only immediately after a young collection. Note that since these objects are allocated directly to old space, there is no cost to promoting them (hence, the subtraction of $B[i]$ ). The differences from before are highlighted with framed boxes. Here a large object allocation is followed by zero or more allocations that fit into young space.

Finally, we modify $f$ :

$$
f[i, j]= \begin{cases}\bar{Y}[i, j]+L[j], & \text { if } L[i]+\bar{Y}[i, j]+\sum_{i \leq k<j} B[k] \leq S_{O} \\ \infty & \text { otherwise. }\end{cases}
$$

This adds the requirement that the promoted data still fits into the old space once the data allocated directly into the old space is included. 
The dynamic program finds the minimum schedule cost, i.e., the minimum cost attained by any legal schedule. More than one schedule may have that cost, of course, but in any case, how can one extract the schedule from the solution, as opposed to just the cost? This is straightforward, and can be done after the fact or as we go. The "as we go" form is easier to explain: As we compute each table entry that has multiple options, we record also which option (which often includes an array index) we used for the minimum cost. Doing this along the way adds a constant factor overhead in space and time. Doing it after the fact requires some scanning, but it is proportional to $n$. For example, to find how $Y[i, j]$ was minimized, we scan values of $k$ seeking to minimize $Y[i, k]+y[k, j]$. This is best done starting from $j-1$ and working backward, stopping either at $i$ or when $y[k, j]$ becomes $\infty$.

\subsection{Complexity Analysis}

We consider the asymptotic complexity of computing each array of our dynamic programming solution in turn. Since the sizes of the arrays are determined by $n$, the number of groups, we use $n$ as our measure of the size of the problem. However, the actual input to the dynamic program is the table of $c[\cdot, \cdot, \cdot]$ values, which leads to the question of how the size of $c$ relates to $n$. As mentioned in Section 4.2, a first thought would be that $c$ could have size $O\left(n^{3}\right)$ because of the possible values for the indices $p, b$, and $d$ of the $c$ table, but in fact the number of cohorts is bounded by the number of objects, which in the worst case is $A / m$, where $m$ is the minimum object size (a constant determined by the language implementer, typically $4,8,16,24$, or 32 bytes). This will be larger than $n^{3}$ only when $G / m>n^{2}$, roughly. To see this, note that $G / m$ is the maximum number of objects per group, and recall that $n$ is the number of groups. This requires traces to be fairly short, and in any case, asymptotically, $O(n)$ still holds. This confirms that $n$ is a sensible measure of the size of the problem-it determines both the size of the dynamic program's arrays and bounds the size of the $c$ table given as input.

$L$ can be computed efficiently with two copies of $c$, one sorted in birth order and the other in death order, so the cost to fill $L$ is at most $O(|c| \log |c|) . A[i, i]=0$ and $A[i, i+1]$ can be determined with one pass over $c$. The remaining $A[i, j]$ values require a total of $O\left(n^{2}\right)$ work to compute. However, we really need the values only for $j-i \leq 2\left(S_{Y} / G\right)$, i.e., if $i$ and $j$ are more than a certain distance apart, $A[i, j]$ necessarily exceeds $S_{Y}$. For a given $i$, we need entries up through the first $j$ that gives $A[i, j]>S_{Y}$, but none after that. Each group adds more than $G / 2$ bytes on average. In the end, we need only $O(n)$ entries of $A[\cdot, \cdot]$, a constant number per row, rather than $O\left(n^{2}\right)$. Given $A$, it is trivial $(O(n))$ to compute $B$.

Now consider $y$. For each element of $c$, we can add it to just those $y$ entries for which it is relevant. Since at most $2 S_{Y} / G$ groups (call this value $g$ ) can occur between $i$ and $j$ for $y[i, j]$ to be finite, $c[p, b, d]$ adds to at most $2 g$ elements of $y$. However, $g$ is a constant, so each element of $c$ incurs constant work for this term. Therefore, the total cost to calculate that term is $O(n)$. A similar argument works for the second term (and the two terms sum two disjoint sets of elements of $c$ ). Since each element of $y$ takes constant time to compute, the total time to compute $y$ is $O\left(n^{2}\right)$. Another way of looking at this is that $y[i, j]$ is necessarily infinite once $j-i$ is large enough. We need fill in only a constant number of diagonals.

$Y$ is computed as the minimum of a number of cases. How many? At most $2 g$ values of $k$ can index elements of $y$ that are not $\infty$, so each $Y$ value is determined by a constant amount of work. Thus, computing $Y$ takes $O\left(n^{2}\right)$ operations. This is where it is important that the second term of the sum is $y$, not $Y$. It might be more natural to instead calculate $\min _{i<k<j} Y[i, k]+Y[k, j]$. But, notice that different choices of $k$ may represent exactly the same underlying schedule of young collections, and would lead to $O\left(n^{3}\right)$ cost to compute $Y$. Since we use $\min _{i<k<j} Y[i, k]+y[k, j]$, each choice of $k$ corresponds to a unique schedule of young collections, and we can "short circuit" 
the computation when $y[k, j]$ becomes infinite. The same argument applies to the calculation of $\bar{Y}$.

Each element of $f$ requires constant time to compute given $Y, L$, and $B$, so its cost is $O\left(n^{2}\right)$. (One does need to precompute $\sum_{0 \leq j \leq i} B[j]$ for each value of $i$ in order to compute the sum term of $f$ efficiently.) Lastly, each element of $F$ requires $O(n)$ time to compute, but there are only $n$ of them, so that cost is $O\left(n^{2}\right)$.

The total cost is dominated by a number of $O\left(n^{2}\right)$ terms, so is $O\left(n^{2}\right)$.

The space required is $O\left(n^{2}\right)$.

Computing in Parallel: Given at least $n$ processors, we can compute in parallel so as to reduce $O\left(n^{2}\right)$ to $O(n)$. However, there are a number of min reductions. The min computations involved for $y, Y$, and $\bar{Y}$ involve at most a constant number of items, so the min does not affect the asymptotic cost. In the case of $f$ and $F$, however, the number of min computations can be $O(n)$ for each element, which requires $O(\log n)$ parallel time. Thus, the asymptotic cost with at least $n$ processors is $O(n \log n)$, and the computation proceeds diagonal by diagonal. If the number of processors, $p$, is less than $n$, the cost will be $O\left(\left(n^{2} / p\right) \log p\right)$.

What are the prospects for exploiting GPUs for this work? The large available $p$ is helpful, but the limits on total memory or on memory bandwidth may be more problematic. We have not worked through all the details, but believe that with some cleverness about which array elements are brought into GPU memory at once, one could achieve high parallelism with GPUs. Still, GPU utilization may be reduced because of memory limitations.

Impact of Varying G: What happens if we vary the group size, $G$ ? Suppose we cut $G$ in half. Then $n$ would approximately double. The number of cohorts might increase some, but it is still bounded by the total number of objects. The most that the number of cohorts could change by is a factor of 8 (each of $p, b$, and $d$ could split into two different values), but this can happen only until we start to approach the number of objects in the trace. The value $g$, our bound on the number of groups that can fit in $S_{Y}$, will double, so various computations' cost will double. So in the worst case, here is the impact on each value's computation: $L$, slightly more than doubled; $A[i, i+1]$ slightly more than doubled; other $A$ values, doubled; $B$, doubled; $y$, each element's cost is doubled (more $c$ values are part of its sum) and twice as many elements are non-infinite, so the cost is quadrupled; $Y$, the cost of each element is doubled, so that the total cost will increase by a factor of $8 ; \bar{Y}$ likewise goes up by a factor of $8 ; f$, quadrupled; and $F$, quadrupled. Thus, the overall cost has a leading term with a factor of about $1 / G^{3}$.

Varying Heap Size: If we change $S_{Y}$ we need to recompute most of the values. However, $S_{O}$ affects only $f$. Therefore, we can compute $y, Y$, and $\bar{Y}$ once for a given $S_{Y}$, and then compute $f$ and $F$ for many values of $S_{O}$ using the same $y, Y$, and $\bar{Y}$ values. $f$ is still $O\left(n^{2}\right)$ to compute, but we certainly save usefully on overall computation time.

What if $G=\boldsymbol{m}$ ? (Recall that $m$ is the minimum object size, so this means considering collection at each object allocation.) If we choose the smallest possible group size, then we are not grouping at all, but considering each object to be its own cohort, and not restricting where collections can occur. The cost to solve the dynamic programming problem may be very high (we typically have used $G=2^{18}$, leading to $n$ 's being a factor of something like 5,000 smaller than the number of objects in the trace). A trickier question is how different is the optimal cost for a given $G$ from the optimal cost for $G=m$. This seems difficult to determine theoretically, though we have not worked hard on the problem. We believe there may be lower bounds we could compute that might in practice show how close the two costs must be. We also develop some empirical evidence of how the optimal cost varies with decreasing $G$, within the memory available to the solver. We summarize all these results in Table 1. 
Table 1. Summary of Complexity Results $\left(^{*}=\right.$ Using a Band Matrix Representation)

\begin{tabular}{|c|c|c|c|c|c|}
\hline Variable & Cost & Impact when $G \rightarrow G / 2$ & When $G=m$ & Parallel & Space \\
\hline \hline$c$ & - & $\sim 2$ & - & - & $O(n)$ \\
\hline$L$ & $O(c \log c)$ & $\sim 2$ & $O(m \log m)$ & $O(\log n)$ & $O(n)$ \\
\hline$A$ & $O(n)$ & $\sim 2$ & $O(m)$ & $O(1)$ & $O(n)^{*}$ \\
\hline$B$ & $O(n)$ & $\sim 2$ & $O(m)$ & $O(1)$ & $O(n)$ \\
\hline$y$ & $O\left(n^{2}\right)$ & $\sim 4$ & $O\left(m^{2}\right)$ & $O(n)$ & $O(n)^{*}$ \\
\hline$Y$ & $O\left(n^{2}\right)$ & $\sim 8$ & $O\left(m^{2}\right)$ & $O(n)$ & $O\left(n^{2}\right)$ \\
\hline $\bar{Y}$ & $O\left(n^{2}\right)$ & $\sim 8$ & $O\left(m^{2}\right)$ & $O(n)$ & $O\left(n^{2}\right)$ \\
\hline$S_{Y}$ terms & $O\left(n^{2}\right)$ & $\sim 8$ & $O\left(m^{2}\right)$ & $O(n)$ & $O\left(n^{2}\right)$ \\
\hline \hline$f$ & $O\left(n^{2}\right)$ & $\sim 4$ & $O\left(m^{2}\right)$ & $O(n \log n)$ & $O\left(n^{2}\right)$ \\
\hline$F$ & $O\left(n^{2}\right)$ & $\sim 4$ & $O\left(m^{2}\right)$ & $O(n \log n)$ & $O(n)$ \\
\hline$S_{O}$ terms & $O\left(n^{2}\right)$ & $\sim 4$ & $O\left(m^{2}\right)$ & $O(n \log n)$ & $O\left(n^{2}\right)$ \\
\hline
\end{tabular}

\section{EXPERIMENTS}

We now describe the experimental methodology we used to assess our GC schedule optimization methods. We begin by describing the general experimental protocols and then summarize the set of program traces that we used in our evaluation.

\subsection{Experimental Protocols}

The primary question of interest in this work is how much better the optimal schedules are than the schedules produced by the corresponding default policies. To answer this question, we evaluate each method on a benchmark suite consisting of multiple programs. Each program can be run with a variety of input files, producing a collection of program traces. The program traces we use are described below.

Each combination of a particular program trace combined with a choice of old and young heap sizes defines a unique problem that we solve using our dynamic programming algorithm. We evaluate the performance of methods for each trace using a range of old heap sizes from zero to five times the maximum live size of the trace, increasing by one block at a time (the group size). For the majority of our results, we use a group (block) size of $256 \mathrm{~KB}$, though we also use smaller blocks in some experiments, as indicated below. We evaluate a number of absolute sizes for the young heap: $1 \mathrm{MB}, 2 \mathrm{MB}, 4 \mathrm{MB}, 8 \mathrm{MB}$, and $16 \mathrm{MB}$.

We compute results for single traces in terms of the mark/cons ratio (traced bytes/allocated bytes) of the default and optimal schedules over the full range of heap values.

\subsection{Traces Used}

We created traces using mostly programs from the DaCapo benchmark suite (version 9.12) [3, 4]. For a number of these programs, namely, avrora, fop, jython, luindex, lusearch, and pmd, we developed additional inputs. Some programs failed to run on our trace generation framework (Elephant Tracks $[10,11]$ ) or for some "sizes" (inputs), particularly tradesoap and larger "sizes" of tomcat and tradebeans, so these traces are omitted from our evaluation. The virtual machine might fail with an internal exception, or the application might deadlock, and so forth.

We added an additional benchmark, javac, which runs the Oracle Java (source to bytecode) compiler. We wanted to synthesize behavior similar to a long-running server program, so we presented the compiler with a substantial number of Java source files to compile and forced it to compile them separately rather than jointly. We further manipulated things so that the compiler 
would release cached soft references between compilations, making it reload information about classes mentioned in each class being compiled-as a server would have to do. We achieved our goal of a highly cyclic pattern of live sizes, growing during each compilation then dropping back to a baseline in between. We believe this server-like benchmark is an interesting case for which to find optimal GC policies. We ran the benchmarks using the IBM Java 6 Java virtual machine.

From the ET traces we extracted object allocation, mutation, and death events, which we consolidated into groups of size $4 \mathrm{~KB}, 16 \mathrm{~KB}, 64 \mathrm{~KB}$, and $256 \mathrm{~KB}$. We computed pre-birth times and cohorts. The cohorts were then used with the dynamic program to find optimal generational collection schedules. As previously mentioned, we used old space sizes from zero to five times the maximum live size (rounded up to a whole block) with points at each whole block in between to develop fairly smooth curves.

Our GC cost measure is the number of bytes traced. As mentioned before, we generally report the "mark/cons" ratio, i.e., bytes traced divided by bytes allocated.

We often compare with a default policy. This policy allocates each group in young space if possible; otherwise it performs a young space collection if occupied young space will fit in old space, and if occupied young space will not fit in the free old space, it performs a full collection. This is a typical policy used in real collectors: allocate until available space is full, then collect. (More generally, actual collectors may choose to adjust the heap size, but as we stated before, that is beyond the scope of the present work.)

Table 2 offers some summary information about each of the traces we used. Since a given program usually has a number of different inputs resulting in a number of different traces, we report sizes by giving the range of values.

\section{RESULTS}

We now report the results of the experiments described in the previous section. We first look at the improvement in cost of optimal schedules compared with those obtained by the default policy, as we vary the heap size. We then look at the empirical cost of running our optimization algorithm. Finally, we explore what happens as we reduce the group size.

\subsection{Heap Size and Collection Cost}

To begin, we consider results for a particular trace: a run of javac on one input, using a young size of $8 \mathrm{MB}$. This trace is chosen because its relatively short length makes various properties of its optimal policies easily visible. ${ }^{14}$ First, Figure 2 shows the points where collections occur in the optimal schedule and under the default policy, using an old size of 25MB. Notice that the young collections in the optimal schedule tend to occur at smaller live sizes than those in the default policy, but they are not always located at exact local minima of the live size curve. This illustrates that minimizing the total cost of a schedule depends on global, rather than simply local, information about the trace. However, also note that the optimal schedule contains two inexpensive full collections during the start-up phase of the program, and only one full collection afterward. This contrasts with the two expensive full collections that the default policy performs, greatly increasing its cost.

Next, Figure 3 plots the cost of optimal and default schedules against the old space size. As expected, the optimal cost is always less than the default cost, and in general costs decrease as the heap size grows larger. However, note that the default cost can sometimes be greater at larger heap sizes, while the optimal cost never increases. This is because at larger heap sizes, at worst, the

\footnotetext{
${ }^{14}$ We make no claim as to whether the heap sizes are what one would choose in practice. This example is chosen only to illustrate how optimal collection can be different from the default policy.
} 
Table 2. Summary of Benchmarks Used

\begin{tabular}{|c|c|c|c|c|c|c|c|}
\hline \multirow[b]{2}{*}{ Benchmark } & \multirow{2}{*}{$\begin{array}{c}\text { \# of } \\
\text { Inputs }\end{array}$} & \multicolumn{2}{|c|}{ Max live (MB) } & \multicolumn{2}{|c|}{ \# of Groups } & \multicolumn{2}{|c|}{ \# of Cohorts } \\
\hline & & $\min$ & $\max$ & $\min$ & $\max$ & $\min$ & $\max$ \\
\hline \multicolumn{8}{|c|}{ 256K Blocks } \\
\hline luindex & 5 & 12900360 & 16033064 & 67 & 211 & 413 & 956 \\
\hline lusearch & 7 & 12554424 & 12585176 & 2780 & 44113 & 7224 & 113589 \\
\hline sunflow & 3 & 13145184 & 15256928 & 444 & 29402 & 1609 & 109898 \\
\hline fop & 14 & 15011488 & 51418376 & 109 & 7160 & 1115 & 41180 \\
\hline avrora & 4 & 13930768 & 22696928 & 203 & 1678 & 1020 & 20238 \\
\hline jython & 7 & 19129104 & 42501680 & 554 & 71981 & 6445 & 181377 \\
\hline pmd & 19 & 13359320 & 96220640 & 83 & 4856 & 713 & 202940 \\
\hline xalan & 3 & 14037792 & 14669112 & 623 & 55076 & 4233 & 321669 \\
\hline javac & 6 & 12114688 & 18529400 & 731 & 14366 & 10354 & 197875 \\
\hline tomcat & 1 & \multicolumn{2}{|c|}{22371048} & \multicolumn{2}{|c|}{1956} & \multicolumn{2}{|c|}{21976} \\
\hline \multicolumn{8}{|c|}{ 64K Blocks } \\
\hline luindex & 5 & 12896824 & 16033056 & 265 & 811 & 1389 & 3245 \\
\hline lusearch & 2 & 12558776 & 12589192 & 16512 & 16563 & 35665 & 38587 \\
\hline sunflow & 2 & 13291384 & 13690752 & 1770 & 41448 & 6094 & 141213 \\
\hline fop & 14 & 15011960 & 51407040 & 439 & 29554 & 3363 & 124806 \\
\hline avrora & 4 & 13957056 & 22696648 & 809 & 6702 & 4328 & 61337 \\
\hline jython & 6 & 19179120 & 42503936 & 2198 & 50297 & 20522 & 210309 \\
\hline pmd & 19 & 13367208 & 96243840 & 325 & 18967 & 2261 & 618627 \\
\hline xalan & 2 & 14074208 & 14109648 & 2646 & 23796 & 13328 & 108851 \\
\hline javac & 6 & 12114456 & 18536568 & 2867 & 55158 & 28324 & 577961 \\
\hline tomcat & 1 & \multicolumn{2}{|c|}{22378784} & \multicolumn{2}{|c|}{7932} & \multicolumn{2}{|c|}{54895} \\
\hline \multicolumn{8}{|c|}{ 16K Blocks } \\
\hline luindex & 5 & 12899696 & 16033128 & 1038 & 3354 & 4480 & 12626 \\
\hline sunflow & 1 & \multicolumn{2}{|c|}{13323040} & \multicolumn{2}{|c|}{7051} & \multicolumn{2}{|c|}{21328} \\
\hline fop & 11 & 15011488 & 46855728 & 1767 & 13860 & 9400 & 74974 \\
\hline avrora & 3 & 13959936 & 19975368 & 3227 & 9651 & 15122 & 57408 \\
\hline jython & 3 & 19199448 & 21083944 & 8742 & 14392 & 55203 & 79118 \\
\hline pmd & 14 & 13381104 & 46402952 & 1325 & 12897 & 6581 & 252763 \\
\hline xalan & 1 & \multicolumn{2}{|c|}{14081432} & \multicolumn{2}{|c|}{9384} & \multicolumn{2}{|c|}{38399} \\
\hline javac & 1 & \multicolumn{2}{|c|}{14370224} & \multicolumn{2}{|c|}{10759} & \multicolumn{2}{|c|}{74842} \\
\hline \multicolumn{8}{|c|}{ 4K Blocks } \\
\hline luindex & 5 & 12907592 & 16035456 & 4604 & 12211 & 12951 & 36862 \\
\hline fop & 6 & 15012600 & 21314000 & 6380 & 13149 & 23655 & 55130 \\
\hline avrora & 2 & 13960944 & 14551648 & 13135 & 24109 & 47615 & 97149 \\
\hline pmd & 6 & 13382160 & 20507384 & 5240 & 17129 & 18455 & 187611 \\
\hline
\end{tabular}

optimal schedule will simply collect at the same points as it will for smaller heap sizes. It may seem counter-intuitive that increasing heap size can cause the default policy to make decisions that cost more. However, this is a (now) well-known effect and comes about because collection points shift and can cause a full collection at a time with high live size shortly before a large number of objects becomes unreachable. Adjusting young collection times can avoid that costly full collection.

As for the optimal schedules, increasing the heap size never excludes schedules that are valid at smaller sizes. Also, note that full collections tend to be much more expensive than young 


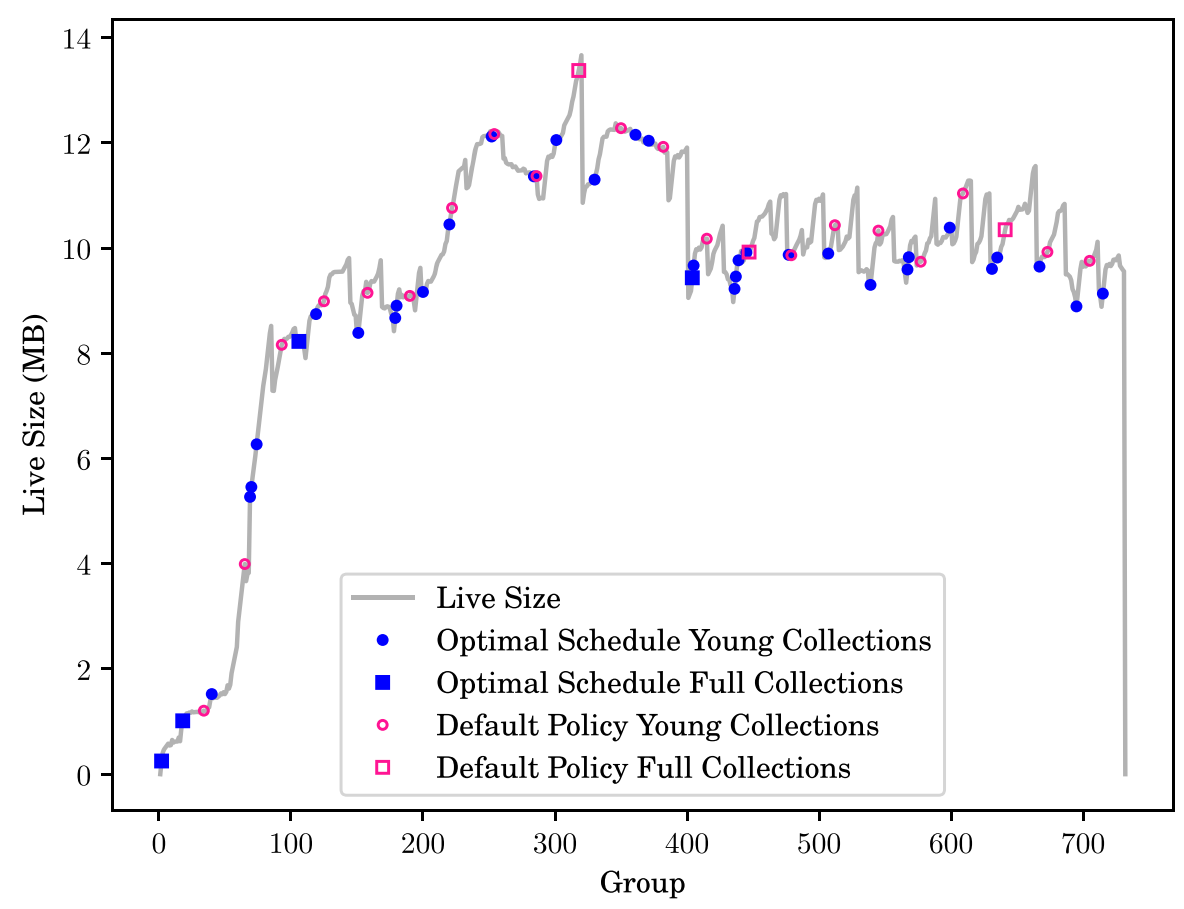

Fig. 2. Collection points for a particular trace.

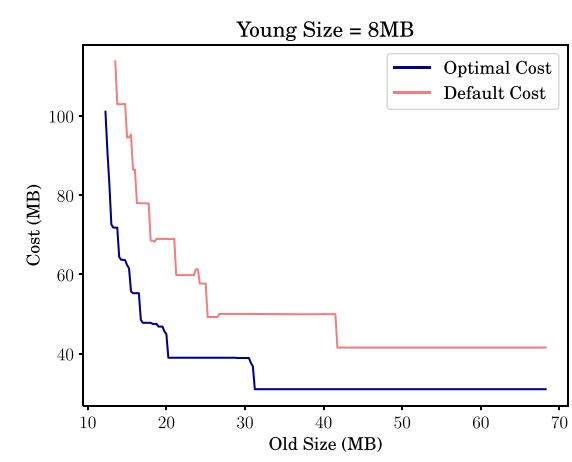

Fig. 3. Cost vs. old size for a particular trace.

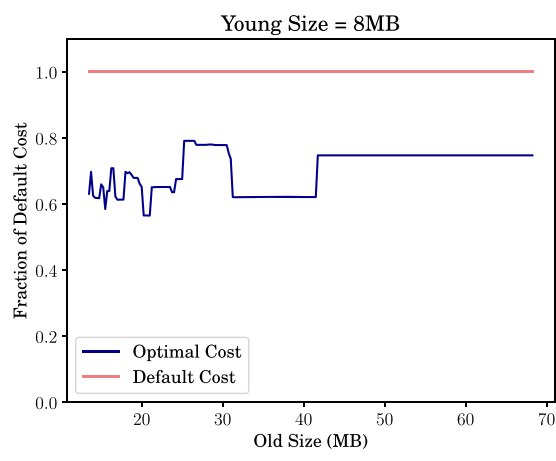

Fig. 4. Scaled cost vs. old size for a particular trace.

collections. As the heap grows, it provides the opportunity for more inexpensive placement of collections. But, when certain thresholds are reached, the trace can complete with one fewer full collection, giving a substantial decrease in costs. This explains the characteristic stair-step shape of the cost curves. The cost decreases slowly until an entire full collection is optimized away, at which point the cost drops quickly. Note, furthermore, that these points occur at smaller old sizes for the optimal schedule than for the default schedule. Finally, notice that the optimal schedule curve extends further left than the default schedule curve. This shows that careful placement of collection points can allow a collector to continue in situations where the default strategy paints itself into a corner with no valid actions left to take. We present the same data again in 


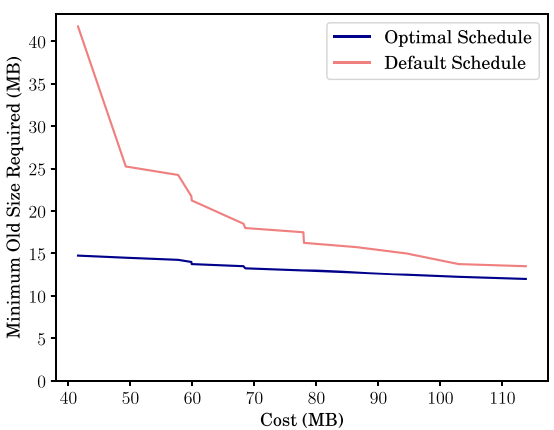

Fig. 5. Min old size vs. cost for a particular trace.

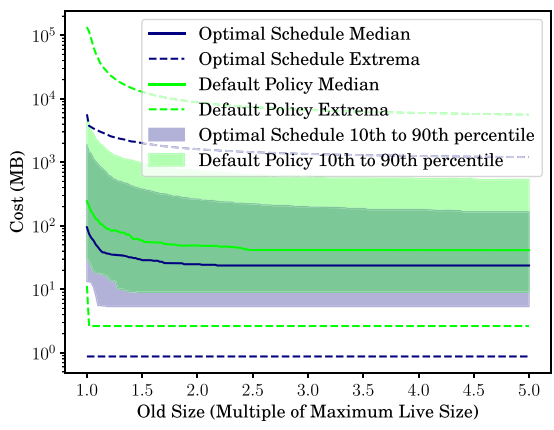

Fig. 7. Overall cost vs. old size.

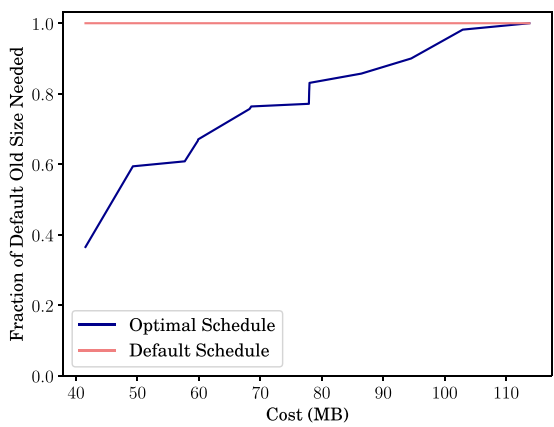

Fig. 6. Scaled min old size vs. cost for a particular trace.

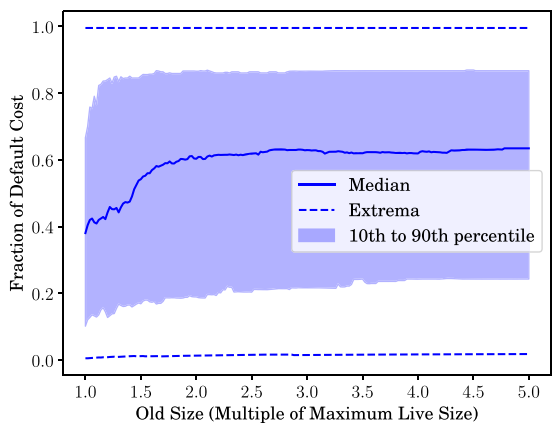

Fig. 8. Overall cost vs. old size, scaled to multiples of the default cost.

Figure 4, scaled by the default schedule cost (at the same heap size). As the old size changes, the relative savings of the optimal schedule varies within certain bounds.

The previous graphs tell us the cost of the two different schedules at various old sizes, but we can ask a different, related question: If one is willing to tolerate a certain cost of collection, what is the smallest old size one can use without exceeding this cost? Figure 5 answers this question. Using the same trace and settings as the previous figures, the horizontal axis shows the cost we are willing to tolerate, and the vertical axis shows the minimum old size needed to meet this requirement. As the cost decreases, the old size needed increases, but it increases much more quickly in the case of the default policy. Figure 6 presents the same data, scaled by the size required by the default schedule. Note that the curves meet in the upper right corner. This shows that if we tolerate a very high cost, we run into the hard limit that the live data must fit into the heap, and we cannot reduce the heap size any more. Essentially, we must collect at nearly every timestep, and no optimization is possible.

Next, we expand our view from a single trace to the cost reductions associated with optimal schedules on a wide range of programs and inputs. Figure 7 shows the relationship between the costs of the default and optimal schedules versus the size of the old space. These results are summaries over all of the programs, inputs, and young sizes we analyzed; in order to draw meaningful comparisons across different traces, the old sizes on the horizontal axis are scaled to be multiples of the maximum volume of live data in the trace ("max live size"). Clearly, the optimal schedule provides a greater cost savings at smaller old sizes, but there is quite a large variance in the data.

Figure 8 shows the same data as Figure 7, but the cost of each optimal schedule has been divided by the cost of the default schedule that corresponds to the same trace and settings. The savings 


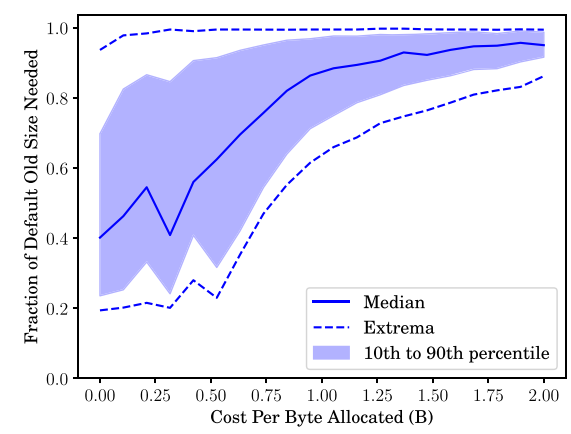

Fig. 9. Overall min old size vs. cost, scaled to multiples of the default size.

become more clearly visible. At larger heap sizes, the average optimal schedule costs just over half what the default schedule does, and the relative cost decreases at smaller old sizes. This matches our intuition that smaller heap sizes are "tighter": they are more sensitive to the placement of collections, and thus provide more opportunity for optimization.

As we did for the single trace, we can ask what minimum old size is needed for a given cost; these results are summarized in Figure 9. In order to compare between traces of different lengths, the horizontal axis is scaled to show the cost per byte allocated, sometimes called the mark/cons ratio. Another, possibly more intuitive, way to describe that quantity is the average number of times an allocated byte will be copied by the collector.

\subsection{Empirical Running Times}

Next, we discuss the running time of our algorithm in practice. Here, we mean the time to find optimal schedules, not the running time of the schedules themselves. Recall that our algorithm can be decomposed into two parts, one which depends only on the young size, and a later part that depends also on the old size. We calculated these parts separately, and thus analyze their running times separately as well. The total running time is, of course, the sum of these two parts. All calculations were run using single-threaded programs on a server with Intel Xeon E5-2690 v3 processors with nominal clock speeds of $2.6 \mathrm{GHz}^{15}$ and cache sizes of $32 \mathrm{~KB}$ (L1 instruction and L1 data), 256KB (L2), and 30MB (L3). We did not record the exact memory usage of our programs, but the system has $256 \mathrm{~GB}$ and main memory, and experience suggested that we were close to this limit for some of the longest traces at block sizes of 256K. Traces with smaller block sizes often exceeded this limit, so those calculations were unable to complete.

First, consider the portion of the algorithm that depends only on the young space size. We fit a model to our data of the form $t=e^{c} \times n^{x_{1}} \times S_{Y}^{x_{2}}$ by taking the log of our input and output and then performing an ordinary least squares regression. This yields $t=e^{-5.82} \times n^{1.12} \times S_{Y}^{1.10}$. Two views of these data are shown in Figures 10 and 11. Note that in Figures 10-17, we have two independent variables, one along the horizontal axis and one represented as the color of our data points. The solid regression line is plotted through the same points. However, since its domain is projected down to a single dimension, it takes on a stair-step appearance: the regression line is vertical in some areas where the data vary only along the "color" dimension. We see that there

\footnotetext{
${ }^{15}$ We allowed the operating system to adjust frequency and voltage as it would normally do. The system was otherwise unused, so this is a realistic execution experience on this platform in the way it would typically be used. We felt this to be the most appropriate approach in this case since we are not, e.g., attempting careful performance comparisons of different versions of an algorithm, but rather trying to analyze what a user's experience of our implementation of this algorithm would be like.
} 


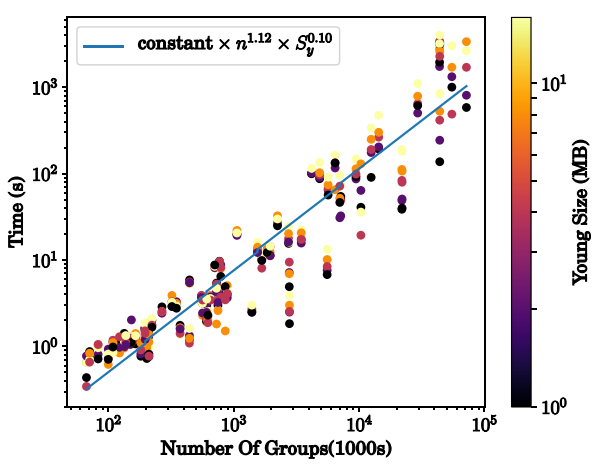

Fig. 10. Relationship between number of groups and calculation time for first part of algorithm.

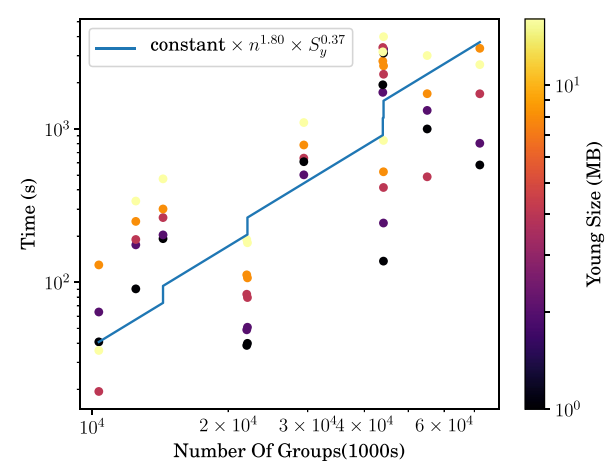

Fig. 12. Relationship between number of groups and calculation time for first part of algorithm, long traces only.

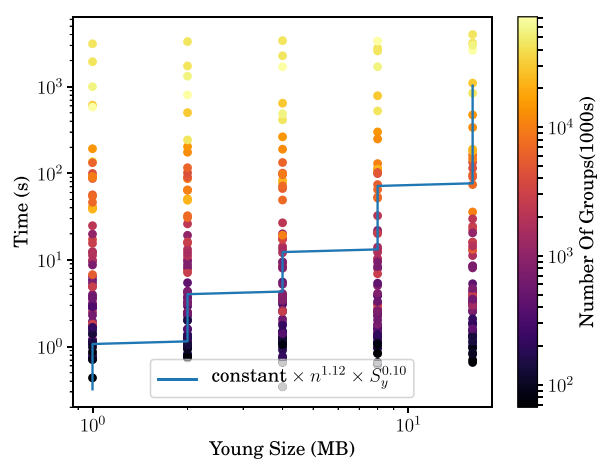

Fig. 11. Relationship between young size and calculation time for first part of algorithm.

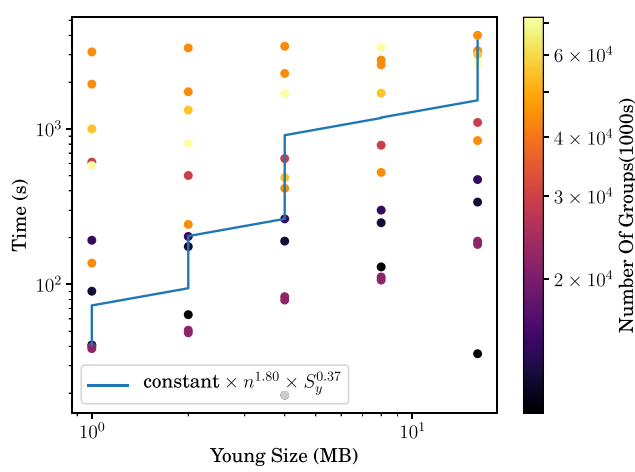

Fig. 13. Relationship between young size and calculation time for second part of algorithm, long traces only.

is more variance in the effect of young size $\left(S_{Y}\right)$ than the effect of the number of groups $(n)$. As we are interested in the asymptotic behavior, we can limit our model to fitting only the traces with 10,000 groups or more. This gives $t=e^{-12.93} \times n^{1.80} \times S_{Y}^{0.37}$, shown in Figures 12 and 13. The exponents show that for longer traces, the dependence on $n$ is nearly $O\left(n^{2}\right)$, while the effect of $S_{Y}$ is sub-linear.

Similarly, for the portion of the algorithm that depends on the old size, we obtain $t=e^{-21.13} \times$ $n^{2.20} \times S_{O}^{0.34}$, as seen in Figures 14 and 15. Again, the variance in the effect of $S_{O}$ is large, and the effect is sub-linear, while the variance for $n$ is small and the effect is strong, greater than $O\left(n^{2}\right)$. Restricting to traces at least 10,000 groups long yields $t=e^{-12.63} \times n^{1.34} \times S_{O}^{0.34}$ (Figures 16 and 17). The dependence on $S_{O}$ is similar, but the effect of $n$ is reduced, super-linear but well below $O\left(n^{2}\right)$, which is good news for practical calculation.

All of the values calculated are highly significant statistically; their p-values are given in Table 3 . Note that zeros do not indicate missing values; they are rounded from values that were too small for the statistical software we used. 


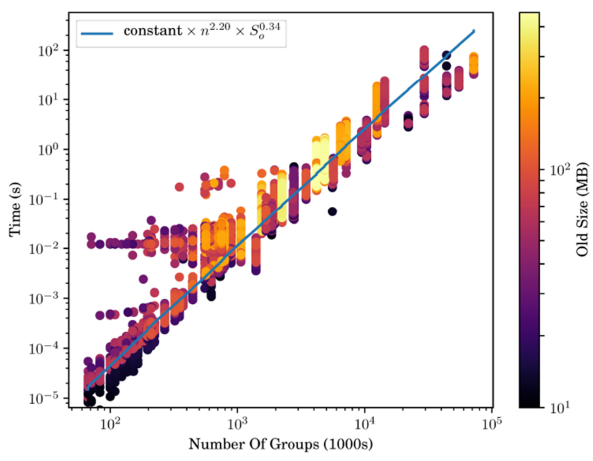

Fig. 14. Relationship between number of groups and calculation time for second part of algorithm.

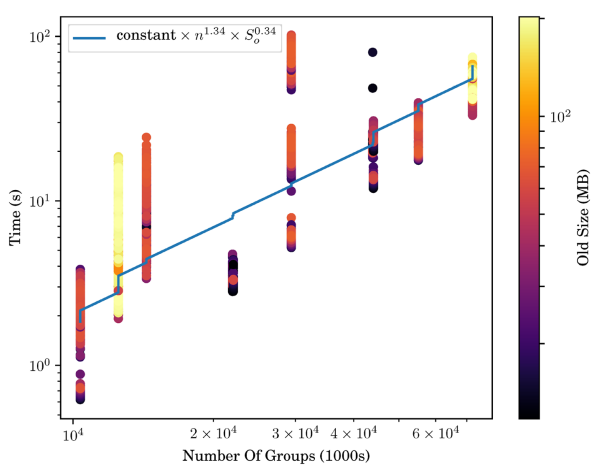

Fig. 16. Relationship between number of groups and calculation time for second part of algorithm, long traces only.

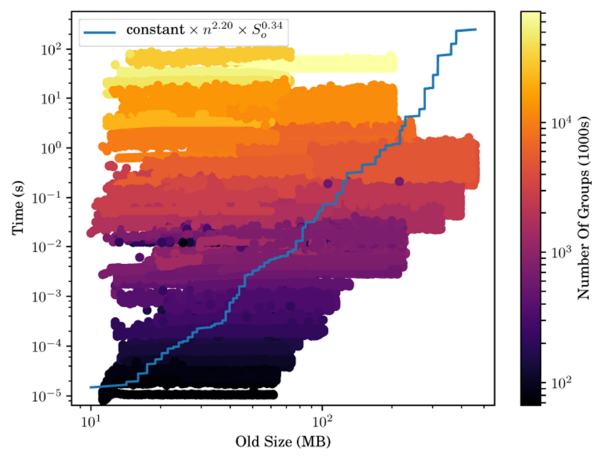

Fig. 15. Relationship between old size and calculation time for second part of algorithm.

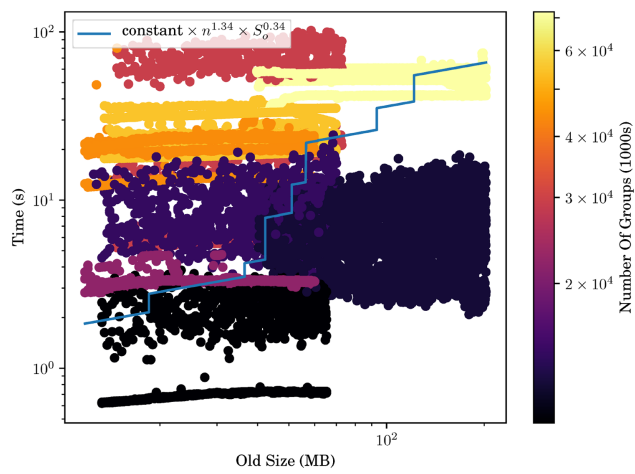

Fig. 17. Relationship between old size and calculation time for second part of algorithm, long traces only.

Table 3. Running Time Regression Model Data $*=Y$ for Young, $O$ for Full

\begin{tabular}{|l|r|r|r|r|}
\hline Variable & $\begin{array}{c}\text { Young Part all } \\
\text { traces }\end{array}$ & $\begin{array}{c}\text { Young Part } \\
\text { long traces }\end{array}$ & $\begin{array}{c}\text { Full Part all } \\
\text { traces }\end{array}$ & $\begin{array}{c}\text { Full Part long } \\
\text { traces }\end{array}$ \\
\hline \hline$R^{2}$ & 0.89 & 0.62 & 0.97 & 0.72 \\
\hline$R^{2}$ Adjusted & 0.89 & 0.61 & 0.97 & 0.72 \\
\hline $\mathrm{F} \mathrm{p}$-value & $2.07 \mathrm{e}-167$ & $1.06 \mathrm{e}-11$ & 0 & 0 \\
\hline AIC & 757 & 151 & $257 \mathrm{e} 3$ & $296 \mathrm{e} 2$ \\
\hline $\mathrm{c}$ & -5.82 & -12.93 & -21.13 & -12.63 \\
\hline $\mathrm{c} \mathrm{t} \mathrm{p-value}$ & $1.99 \mathrm{e}-118$ & $1.20 \mathrm{e}-7$ & 0 & 0 \\
\hline $\mathrm{c}$ standard error & 0.16 & 2.11 & 0.01 & 0.08 \\
\hline $\mathrm{n}$ & 1.12 & 1.80 & 1.34 & 1.34 \\
\hline $\mathrm{n}$ t p-value & $1.38 \mathrm{e}-168$ & $7.37 \mathrm{e}-12$ & 0 & 0 \\
\hline $\mathrm{n}$ standard error & 0.021 & 0.21 & $1 \mathrm{e}-3$ & 0.01 \\
\hline$S_{*}$ & 0.11 & 0.37 & 0.34 & 0.34 \\
\hline$S_{*} \mathrm{t} \mathrm{p}$-value & $8.11 \mathrm{e}-3$ & $6.07 \mathrm{e}-3$ & 0 & 0 \\
\hline$S_{*}$ standard error & 0.04 & 0.13 & $2 \mathrm{e}-3$ & 0.01 \\
\hline
\end{tabular}




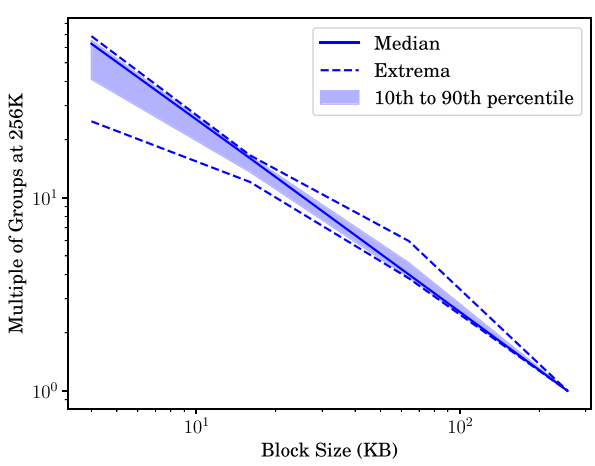

Fig. 18. Group size vs. number of groups.

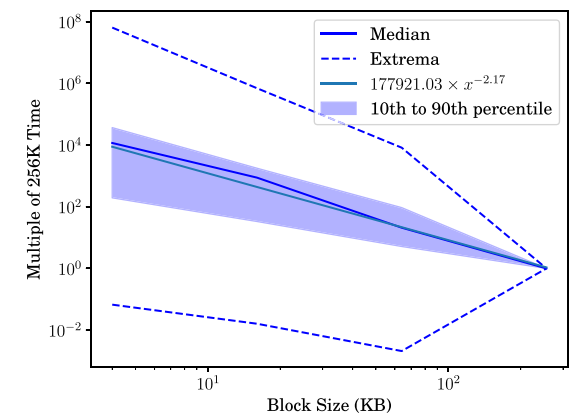

Fig. 20. Group size vs. time to calculate optimal schedule, first part of algorithm.

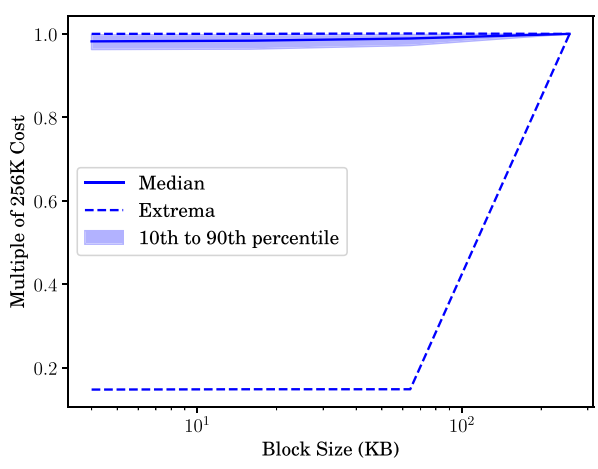

Fig. 19. Group size vs. cost of optimal schedule.

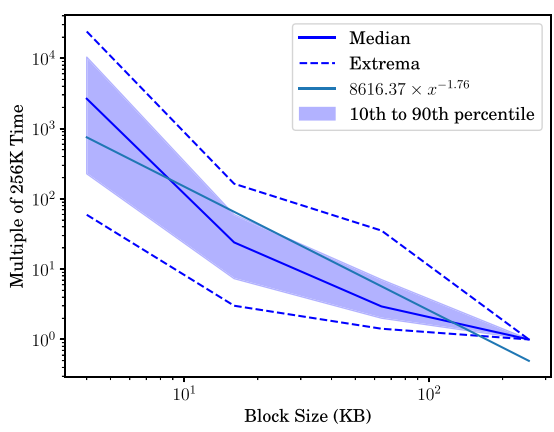

Fig. 21. Group size vs. time to calculate optimal schedule, second part of algorithm.

\subsection{Effect Of Group Size}

As discussed, in our calculations we use groups of size $256 \mathrm{~K}$ and limit collections to groups boundaries. In this section, we will justify this choice.

First, Figure 18 shows the relationship between the group size used and the number of groups in the resulting trace. The relationship is nearly linear: halving the size of a group approximately doubles the number of groups in the trace. Next, Figure 19 shows the effect of the group size on the cost of the optimal schedule. Note that calculating the optimal schedules for most traces at small group sizes exceeds our computational resources. Therefore, we average only over traces for which we have results at all group sizes. These are listed in the $4 \mathrm{~K}$ section of Table 2. Furthermore, to find a truly optimal schedule, we would have to allow for the possibility of a collection after each object is allocated. However, reducing the group size from $256 \mathrm{~K}$ to $4 \mathrm{~K}$ reduces the cost of the optimal schedule by about $2 \%$ in the majority of cases. In a small number of cases, quite large cost reductions are possible. This occurs when an optimal schedule at a smaller group size contains one fewer full collection than an optimal schedule at a larger group size. However, this effect is relatively rare, and depends so sensitively on the exact trace and configuration of the collector that we expect it not to be important in practice.

Finally, Figures 20 and 21 show the relationship between the group size and the time to calculate the optimal schedule. Again, we average only over traces the traces in the $4 \mathrm{~K}$ section of Table 2 
for which we have results at all group sizes. Clearly, the time increases very quickly as the group size decreases $\left(O\left(n^{2.17}\right)\right)$. This is a factor of about 4.5 for each halving of the group size, or 150 for each 10-fold reduction. Overall, this shows that group size has a small impact on the cost of the resulting optimal schedule, but a large impact on the computational resources needed to compute the schedule. This clearly motivates the need for a group-based model of the collector for these analyses.

\section{CONCLUSIONS}

We believe this is the first work to determine provably optimal GC costs (in terms of bytes traced) for runs of real programs. We accomplished this in a specific, but realistic, block-oriented generational collector design. A second contribution is the introduction of pre-birth time, which allows an up-front analysis of the object graph, allocation, and death times to enable rapid determination of when dead young space objects will be promoted. Further, it enables, in a block-oriented scheme, the grouping of many objects together into cohorts that will always act the same in collectors that collect in age order (older objects no later than younger ones). Together, cohorts and blocks greatly speed determination of the number of bytes traced for a given GC schedule. A third contribution is formulation of both NG and Gen GC costs as state machines in terms of pre-birth, birth, and death times of objects (or groups). A fourth contribution is the determination of optimal Gen GC schedule costs (and by implications, schedules) via dynamic programming (which also works for NG GC). A fifth contribution is extension of the two-generation Gen GC dynamic program to an arbitrary number of generations (and other generalizations (see Appendix B)). Finally, we offer a number of empirical results computed from traces of Java programs running various inputs, mostly from the DaCapo benchmark suite. These results show that there is room for program-specific GC policies to reduce GC cost consistently. Program-specific GC policies appear worthwhile for some programs, while for others the impact on the total performance of an application may not be worth the effort. Perhaps of more significance is the result that optimal schedules allow a program to execute in substantially smaller space with the same time overhead as default GC schedules. Also, we observed that optimized performance varies more smoothly with heap size than does performance with a default policy. This smoother tradeoff may be important for some kinds of systems.

One future direction is to use machine learning methods to develop generalizable programspecific GC policies. These might be based on limited, focused instrumentation of a given program. Knowing the optimal schedule can help in training a machine learning algorithm, by giving feedback as to how a learned policy compares with optimal performance. Our dynamic program may also make it easier to obtain good, but less than optimal, schedules for broader training of a machine learning model.

Another future direction is to remove some of the particularities and restrictions involved in our method. For example, perhaps we can bound the difference between optimal cost for a given group size and optimal cost with no group size restriction. Also, modern systems run much larger, longer-running programs. It would be a significant contribution to devise ways to apply these results to such systems. One might also investigate whether the dynamic programming approach can be used to solve optimal collection under different cost models, as sketched in Appendix B. 


\section{APPENDIXES}

\section{A SYMBOLS DEFINED}

Table 4. Table of Symbols

\begin{tabular}{|c|c|}
\hline Name & Description \\
\hline $\bar{n}$ & $\begin{array}{l}\text { The number of time steps in a trace, equal to the total number of blocks (groups) } \\
\text { allocated in the trace }\end{array}$ \\
\hline$P$ & The program performing the allocation \\
\hline$H$ & The heap, or the total heap size \\
\hline$C$ & The controller \\
\hline$t$ & The timestep, the number of blocks or objects that have been allocated before this point \\
\hline$b(x)$ & The birth time of object $x$ \\
\hline$d(x)$ & The death time of object $x$ \\
\hline$s(x)$ & The size in bytes of object $x$ \\
\hline$p(x)$ & The pre-birth time of object $x$ \\
\hline$\Omega$ & The set of all objects allocated during a program's execution \\
\hline$L_{t}$ & The volume of data live at time $t$ in the heap of an NG collector \\
\hline$D_{t}$ & The volume of data dead at time $t$ in the heap of an NG collector \\
\hline$F_{t}$ & The amount of free space in the heap of an NG collector at time $t$ \\
\hline$S$ & The total size of the heap of an NG collector \\
\hline$S_{O}$ & The size of the old space of a Gen collector \\
\hline$S_{Y}$ & The size of the young space of a Gen collector \\
\hline$\pi$ & A policy, a mapping from the visible state of a collector to one of the available actions \\
\hline $\mathcal{A}$ & The set of all actions that are possible for a collector \\
\hline $\mathcal{A}_{t}$ & The subset of actions that are available at time $t$ \\
\hline$V_{t}$ & The visible state of a collector at time $t$ \\
\hline$A_{t}$ & The total amount of space that has been allocated at time $t$ in an NG collector \\
\hline$X_{t}$ & Information available at time $t$ from instrumenting the system \\
\hline$a_{t}$ & The particular action chosen at time $t$ \\
\hline$\delta_{t}$ & The set of time points whose objects were announced as dead \\
\hline$\sigma_{t}$ & The total number of bytes of all objects that die at timestep $t$ \\
\hline$L_{t}^{Y}$ & The volume of data that is live in the young space of a generational collector at time $t$ \\
\hline$L_{t}^{O}$ & The volume of data that is live in the old space of a generational collector at time $t$ \\
\hline$D_{t}^{Y}$ & The volume of data that is dead in the young space of a generational collector at time $t$ \\
\hline$D_{t}^{O}$ & The volume of data that is dead in the old space of a generational collector at time $t$ \\
\hline$F_{t}^{Y}$ & The amount of free space in the young space of a generational collector at time $t$ \\
\hline$F_{t}^{O}$ & The amount of free space in the old space of a generational collector at time $t$ \\
\hline$B_{t}$ & The volume of baggage at time $t$ \\
\hline$T_{t}^{Y}$ & The timestep of the young collection previous to $t$ \\
\hline$T_{t}^{O}$ & The timestep of the full collection previous to $t$ \\
\hline$\delta_{t}^{O}$ & $\begin{array}{l}\text { The set of indices of all objects that die at time } t \text {, and were born before the last young } \\
\text { collection }\end{array}$ \\
\hline
\end{tabular}

(Continued) 
Table 4. Continued

\begin{tabular}{|c|l|}
\hline Name & \\
\hline \hline$\sigma_{t}^{O}$ & The total volume of live objects to be promoted at time $t$ \\
\hline$\delta_{t}^{B}$ & The indices of all objects that are baggage at time $t$ \\
\hline$\sigma_{t}^{B}$ & The total volume of baggage at time $t$ \\
\hline$g$ & The number of groups in a trace \\
\hline$G$ & The size of a group in bytes \\
\hline$m$ & The minimum size of an object in bytes \\
\hline$s$ & The average object size in bytes \\
\hline$L[t]$ & The volume of data live at time $t$ \\
\hline$c[p, b, d]$ & The size in bytes of the cohort that is pre-born at $p$, born at $b$, and dies at $d$ \\
\hline$A[i, j]$ & The total volume of data allocated between $i$ and $j$ \\
\hline$y[i, j]$ & The cost of a young collection at $j$, given that the previous collection was at $i$ \\
\hline$Y[i, j]$ & $\begin{array}{l}\text { The minimum cost of a schedule that begins at } i, \text { ends with a young collection at } j, \text { and } \\
\text { contains only young collections }\end{array}$ \\
\hline $\bar{Y}[i, j]$ & $\begin{array}{l}\text { The minimum cost of a schedule that begins at } i, \text { ends at } j \text {, and contains only young } \\
\text { collections }\end{array}$ \\
\hline$f[i, j]$ & $\begin{array}{l}\text { The minimum cost of a schedule that begins at } i \text {, contains only young collections from } i \\
\text { to } j-1 \text {, and ends with a full collection at } j\end{array}$ \\
\hline$F[j]$ & The minimum cost of a schedule that begins at 0 and ends with a full collection at $j$ \\
\hline$B[i]$ & The size of the group allocated at $i$ if it is bigger than the young space, and 0 otherwise \\
\hline$p$ & The number of processors available for parallel computations \\
\hline$\Pi[i, j]$ & The amount promoted from a collection at $j$, given the previous collection was at $i$ \\
\hline$K$ & The number of generations in a multi-generation scheme \\
\hline$\gamma$ & A generation of a multi-generation scheme \\
\hline$S_{1}, \ldots$ & Size of a generation in a multi-generation scheme \\
\hline$y_{1}, \ldots$ & Multi-generational analog of $y$ \\
\hline$Y_{1}, \ldots$ & Multi-generational analog of $Y$ \\
\hline $\bar{Y}_{1}, \ldots$ & Multi-generational analog of $\bar{Y}$ \\
\hline$\tau[i, j]$ & Time that passes from $i$ to $j$ in a trace \\
\hline & \\
\hline
\end{tabular}

\section{B EXTENDED DYNAMIC PROGRAM FOR OPTIMAL GC SCHEDULES}

We present here a significant generalization of the Gen GC dynamic program of Section 5.2. ${ }^{16}$ Essentially it adds the ability to explore tradeoffs in space as well as time of collection. This enables the development of a scheme that can handle, among other things, any number of fixed-size generations. It is that case that we develop in detail, and then we sketch how the equations can be used to solve some related optimal GC schedule problems.

We assume cohorts $c[p, b, d]$ as in the main article. We observe that if, for a given generation, a collection occurs at $i$ and the next collection is at $j>i$, then the volume of objects promoted can be defined using only knowledge of $c$. This was used in the equations of Section 5.2, but we find it convenient to give that quantity a name here, $\Pi$ :

$$
\Pi[i, j]= \begin{cases}\sum_{i \leq b<j \wedge j \leq d} & c[p, b, d] \text { (live) } \\ +\quad \sum_{p<i \wedge i \leq b \wedge d<j} & c[p, b, d] \text { (baggage) } .\end{cases}
$$

\footnotetext{
${ }^{16}$ We are grateful to an anonymous reviewer for inspiring us to explore this extension.
} 
We also reuse the definition of $A[i, j]$, giving it again for convenience:

$$
A[i, j]=\sum_{i \leq b<j} c[p, b, d] .
$$

We assume a series of generations numbered $1,2, \ldots, K$, and will use the symbol $\gamma$ to range over generation numbers. We further assume generation sizes $S_{1} \leq S_{2} \leq \cdots \leq S_{K}$, generalizing $S_{Y}$ and $S_{O}$.

We now develop space-constrained versions of $y$, and so forth, starting with $y_{\gamma}\left[i, j, S, S^{\prime}\right]$. We desire that to mean the lowest cost collection schedule at generation $\gamma$ (and below) that

(1) has a $\gamma$ collection at $i$ (whose cost is not included in $y_{\gamma}\left[i, j, S, S^{\prime}\right]$ );

(2) has a $\gamma$ collection at $j$ (whose cost is included in $y_{\gamma}\left[i, j, S, S^{\prime}\right]$ );

(3) where promotion into $\gamma$ does not exceed $S$; and

(4) promotion out of $\gamma$ does not exceed $S^{\prime}$.

If either space constraint is exceeded, $y_{\gamma}\left[i, j, S, S^{\prime}\right]$ will be $\infty$, meaning the configuration is infeasible. Here is a suitable definition for the base case, $y_{1}$ :

$$
y_{1}\left[i, j, S, S^{\prime}\right]=\Pi[i, j] \text { if } A[i, j] \leq S \wedge \Pi[i, j] \leq S^{\prime} ; \infty \text { otherwise. }
$$

This captures the first size constraint from $A[i, j]$ fitting into $S$. The amount promoted into generation 2 is $\Pi[i, j]$, and that volume promoted is also the cost in this case. We will be interested for now only in the case of $S=S_{1}$, but as we will see, $S^{\prime}$ may vary.

Here is the general case for $\gamma>1$ (though it refers to $\bar{Y}$, defined below):

$$
y_{\gamma}\left[i, j, S, S^{\prime}\right]=\bar{Y}_{\gamma-1}\left[i, j, S_{\gamma-1}, S\right] \text { if } \Pi[i, j] \leq S^{\prime} ; \infty \text { otherwise. }
$$

We next define $Y_{\gamma}\left[i, j, S, S^{\prime}\right]$, analogous to $Y$ of the Gen GC dynamic program, which allows multiple $\gamma$ collections between $i$ and $j$, but still assumes one at $j$. It will enforce the same space constraints as for $y_{\gamma}$. Here is its equation:

$$
Y_{\gamma}\left[i, j, S, S^{\prime}\right]=\min \left\{\begin{array}{l}
y_{\gamma}\left[i, j, S, S^{\prime}\right], \\
i<k<j \wedge 0 \leq \sigma \leq S^{\prime}
\end{array} Y_{\gamma}\left[i, k, S, S^{\prime}-\sigma\right]+y_{\gamma}[k, j, S, \sigma] .\right.
$$

The first term of the min handles the case of no $\gamma$ collections between $i$ and $j$, and the second handles the case of one or more collections between $i$ and $j$, where the last one before $j$ is at $k$. Notice that the cost is a sum of costs, but that we also explore a space dimension with $\sigma$. This new variable allows the promotion out of $\gamma$ to be split between the $Y$ and $y$ sub-terms, but still sum to no more than $S^{\prime}$. As with $y_{1}$, we are interested only in $S=S_{\gamma}$, but we now see why $S^{\prime}$ may vary: because of the choice of $\sigma$. (If one were to implement the algorithm, it may help a little to recognize that $\sigma$ must be at least $\Pi[k, j]$ for feasibility. It is also possible to develop equations for the minimum possible promotion for $Y_{\gamma}\left[i, k, S, S^{\prime}-\sigma\right]$, which might bound the min computation more quickly in practice.)

In general, $k$ may take on up to $n$ values, i.e., it ranges over the whole trace, though in practice the space constraints will tend to narrow that range. The worst case is $n$, however, because the survival (promotion) rate can be arbitrarily low, allowing $\gamma+1$ collections to be arbitrarily far apart. On the other hand, $\sigma$ may take on at most $S_{\gamma+1}$ values. Asymptotically, we can view that as a constant, though in practice this extra dimension of exploration is likely to be large.

As for Gen GC, we extend $Y_{\gamma}$ to $\bar{Y}_{\gamma}$ :

$$
\bar{Y}_{\gamma}\left[i, j, S, S^{\prime}\right]=\min _{\left\{k \mid i \leq k \leq j \wedge \bar{Y}_{\gamma-1}\left[k, j, S_{\gamma-1}, S\right]<\infty\right\}} Y_{\gamma}\left[i, k, S, S^{\prime}\right] .
$$


As with $y_{\gamma}$, this brings in the space available in the next younger generation, $S_{\gamma-1}$. As long as we have a suitable value to offer in the expression $\bar{Y}_{\gamma-1}\left[i_{1}, i_{2}, \cdot, S\right]$ for a given $i_{1}, i_{2}$, and $S$, this formulation works, but for our extension to just the K-generation case, $S_{\gamma-1}$ is appropriate.

For the base case we define $\bar{Y}_{0}$ as

$$
\bar{Y}_{0}\left[i, j, S, S^{\prime}\right]=A[i, j] \text { if } A[i, j] \leq S^{\prime} ; \infty \text { otherwise. }
$$

This is the space that allocation from $i$ to $j$ will consume in the youngest generation (generation 1). Here $S$ is meaningless since there is no younger generation, so the equation ignores it.

Concerning $\bar{Y}_{\gamma}$, the cost is similar to $\bar{Y}$ of Gen GC, but here the constraint requires that the promotion from $\gamma-1$ over $k$ through $j$ fit in $S_{\gamma}$.

The key thing that we have done is to break apart the cost minimization from the consideration of feasibility within the spaces allowed. Our use of $\sigma$ to give the $Y$ and $y$ parts of the $Y$ equation space constraints different from the cost decouples the two, at the expense of adding a dimension of exploration in the minimization.

The highest generation, $K$, is where full heap collection can occur. We generalize the $f$ equation of Section 5.2 to

$$
f[i, j]=\bar{Y}_{K-1}\left[i, j, S_{K-1}, S_{K}-L[i]\right]+L[j] .
$$

Here, $\bar{Y}_{K-1}$ enforces the space constraint by being $\infty$ if the constraint is infeasible. $F$ is defined as before.

We omit more complex equations to handle objects larger than a space $S_{i}$, but believe that an approach similar to that of Section 5.2 will also work here.

Complexity: As mentioned above, the minimizations over $k$ in $Y_{\gamma}$ and $\bar{Y}_{\gamma}$ inject a cost factor of $O(n)$, and the minimization over $\sigma$ in $Y_{\gamma}$ injects a cost factor of $O(H)$. In the worst case $i$ and $j$ range over $O(n)$ values, while $S$ is fixed at $S_{\gamma}$ and $S^{\prime}$ ranges over up to $O(H)$ values. Thus, the total cost for one of the arrays is $O\left(n^{2} H\right)$ entries times $O(n H)$ cost per entry, giving $O\left(n^{3} H^{2}\right)$.

We believe the formulation above can be used to create other extensions of the Gen GC model of Section 5.2:

Large Object Spaces: A large object space is a space set aside where large, non-pointercontaining objects can be stored and managed without copying. These speed the handling of large binary data. The issue with our previous formulation is that such objects have no promotion cost (since they are not copied), but they logically consume space in the generation to which they currently belong. Our first formulation required that cost equal volume promoted. The extended formulation of this appendix allows the two to be separated, so the volume of such large objects residing in a generation can be subtracted from the cost, while the space constraints would remain the same.

Time-Varying Space Budgets: To this point we have considered only schemes where the size of each generation is fixed. The formulation above may allow total space allocation to be traded off across different generations, finding the best fixed generation-size configuration from a total space budget, or even trading off between different generations dynamically, as in Appel's collection system [1]. Given a firm upper bound, one can also explore costs in a regime that grows and shrinks the total space used.

Other Cost Models: For the equations above to work properly to find the minimum cost schedule, the following properties are required:

- Cost must be non-increasing as we increase $S$ or $S^{\prime}$, i.e., allowing more space cannot increase the cost. 
- The added cost of a collection at a given level $\gamma$ at time $j$, given that the previous $\gamma$ collection was at $i<j$, needs to be independent of the pattern of collections at lower levels.

An interesting example cost model is to include a space rental charge for the occupied part of each generation. Here, one can use various definitions of time, as long as the time interval from $i$ to $j, \tau[i, j]$, is defined only in terms of $i$ and $j$ and is non-increasing with $i$ and non-decreasing with $j$. Thus, one could use the number of groups, object, or bytes allocated as a measure of time. If one knew the real or virtual times at which objects were allocated in terms of execution time, number of instructions executed, and so forth, one could use those. Undoubtedly other cost models might also be of interest.

Multiple Collections Before Promotion: Some generational collection algorithms hold objects in a given generation until they have survived multiple collections. It may be possible to model that with the approach given here, possibly by modeling the different age groups in a generation as separate generations, but forcing them to be collected always at the same time.

Out-of-Order Collection: All the models we describe so far require objects be collected, at any given level, in the order in which they are allocated. It might be possible to generalize the model of this appendix to allow allocation directly into older generations without requiring younger generations to be collected first. Likewise, it might be possible to decouple further collections of each space or generation. The challenge in that case is that the equations for each "generation" (space) $\gamma$ might depend on previous collection patterns of all the spaces, not just the spaces numbered lower than $\gamma$. This may blow up the number of dimensions of search considerably and might therefore be of only theoretical interest. The coupling of various spaces together might also "break" the logic of $\Pi$ and the definition of cohorts, so these extensions may require deeper adjustment to the ideas behind our dynamic programs.

Of course, there may be further extensions that have not occurred to us as of this writing.

\section{B.1 Mapping to the Gen GC Case}

For clarity, what is it that makes the Gen GC case simpler? In Equation (27), the cost, $\Pi[i, j]$ is the same as the space constraint. Propagating that information into Equation (29), we can determine several facts. First, that $y_{1}[k, j, S, \sigma]$ does not depend on $S$ (this is special to generation 1 ). Second, that if $\sigma<A[k, j], y_{1}$ will be $\infty$. Third, that if $\sigma>A[k, j], y_{1}$ is $\Pi[k, j]$, independent of $\sigma$. Thus, the only interesting value of $\sigma$ is $A[k, j]$. Further, since cost equals promotion, the promotion of $Y_{\gamma}[i, k, \cdot, \cdot]$ is the same as its value, so the constraint can be computed in terms of the value. In sum, we can rewrite the space constraint in terms of the cost values, and need to vary only $k$. Hence, we can drop $S$ and $S^{\prime}$ from the equation. It is interesting that two generations are simpler to optimize than more than two, but that more than three incurs no additional factors of $n$ or $H$.

\section{REFERENCES}

[1] Andrew W. Appel. 1989. Simple generational garbage collection and fast allocation. Software: Practice and Experience 19, 2 (1989), 171-183. DOI : https://doi.org/10.1002/spe.4380190206

[2] Anna Bendersky and Erez Petrank. 2012. Space overhead bounds for dynamic memory management with partial compaction. ACM Transactions on Programming Languages and Systems 34, 3 (2012), 13.

[3] Stephen M. Blackburn, Robin Garner, Chris Hoffmann, Asjad M. Khan, Kathryn S. McKinley, Rotem Bentzur, Amer Diwan, Daniel Feinberg, Daniel Frampton, Samuel Z. Guyer, Martin Hirzel, Antony L. Hosking, Maria Jump, Han Bok Lee, J. Eliot B. Moss, Aashish Phansalkar, Darko Stefanovic, Thomas VanDrunen, Daniel von Dincklage, and Ben Wiedermann. 2006. The DaCapo benchmarks: Java benchmarking development and analysis. In Proceedings of 
the 21st Annual ACM SIGPLAN Conference on Object-Oriented Programming, Systems, Languages, and Applications, OOPSLA 2006, Peri L. Tarr and William R. Cook (Eds.). ACM, 169-190. DOI : https://doi.org/10.1145/1167473.1167488

[4] Stephen M. Blackburn, Kathryn S. McKinley, Robin Garner, Chris Hoffmann, Asjad M. Khan, Rotem Bentzur, Amer Diwan, Daniel Feinberg, Daniel Frampton, Samuel Z. Guyer, Martin Hirzel, Antony L. Hosking, Maria Jump, Han Lee, J. Eliot B. Moss, Aashish Phansalkar, Darko Stefanovic, Thomas VanDrunen, Daniel von Dincklage, and Ben Wiedermann. 2008. Wake up and smell the coffee: Evaluation methodology for the 21st century. Communications of the ACM 51, 8 (2008), 83-89. DOI : https://doi.org/10.1145/1378704.1378723

[5] Hans-Juergen Boehm. 2004. The space cost of lazy reference counting. In Proceedings of the 31st ACM SIGPLANSIGACT Symposium on Principles of Programming Languages (POPL'04), Neil D. Jones and Xavier Leroy (Eds.). ACM, 210-219. DOI : https://doi.org/10.1145/964001.964019

[6] Barry Hayes. 1991. Using key object opportunism to collect old objects. In Proceedings of the ACM Conference on Object-Oriented Programming Systems, Languages, and Applications (ACM SIGPLAN Notices 26 (11)). ACM, 33-46. DOI : https://doi.org/10.1145/117954.117957

[7] Matthew Hertz, Stephen M. Blackburn, J. Eliot B. Moss, Kathryn S. McKinley, and Darko Stefanovic. 2006. Generating object lifetime traces with Merlin. ACM Transactions on Programming Languages and Systems 28, 3 (2006), 476-516. DOI : https://doi.org/10.1145/1133651.1133654

[8] Nicholas Jacek, Meng-Chieh Chiu, Benjamin M. Marlin, and Eliot Moss. 2016. Assessing the limits of programspecific garbage collection performance. In Proceedings of the 37th ACM SIGPLAN Conference on Programming Language Design and Implementation (PLDI'16), Chandra Krintz and Emery Berger (Eds.). ACM, 584-598. DOI: https:// doi.org/10.1145/2908080.2908120

[9] Pengcheng Li, Chen Ding, and Hao Luo. 2014. Modeling heap data growth using average liveness. In International Symposium on Memory Management (ISMM'14), David Grove and Samuel Z. Guyer (Eds.). ACM, New York, 71-82. DOI : https://doi.org/10.1145/2602988.2602997

[10] Nathan P. Ricci, Samuel Z. Guyer, and J. Eliot B. Moss. 2011. Tool demonstration: Elephant tracks-Generating program traces with object death records. In Proceedings of the 9th International Conference on the Principles and Practice of Programming in fava. ACM, 39-43.

[11] Nathan P. Ricci, Samuel Z. Guyer, and J. Eliot B. Moss. 2013. Elephant tracks: Portable production of complete and precise GC traces. In International Symposium on Memory Management (ISMM'13), Perry Cheng and Erez Petrank (Eds.). ACM, 109-118. DOI : https://doi.org/10.1145/2464157.2466484

[12] J. M. Robson. 1971. An estimate of the store size necessary for dynamic storage allocation. Fournal of the ACM 18, 3 (July 1971), 416-423. DOI : https://doi.org/10.1145/321650.321658

[13] J. M. Robson. 1974. Bounds for some functions concerning dynamic storage allocation. fournal of the ACM 21, 3 (July 1974), 419-499. DOI : https://doi.org/10.1145/321832.321846

[14] J. M. Robson. 1980. Storage allocation is NP-hard. Information Processing Letters 11, 3 (Nov. 1980), 119-125. DOI: https://doi.org/10.1016/0020-0190(80)90124-6

Received March 2018; revised August 2018; accepted August 2018 